\title{
Responses to Glossopharyngeal Stimulus in the Early Embryonic Chick Brainstem: Spatiotemporal Patterns in Three Dimensions from Repeated Multiple-Site Optical Recording of Electrical Activity
}

\author{
Katsushige Sato, Yoko Momose-Sato, Tetsuro Sakai, Akihiko Hirota, and Kohtaro Kamino \\ Department of Physiology, Tokyo Medical and Dental University School of Medicine, Bunkyo-ku, Tokyo 113, Japan
}

In an effort to assess the spatial patterning of glossopharyngeal responses in the early embryonic chick brainstem, we used a multiple-site optical recording system with a $12 \times 12$ element photodiode array and a voltagesensitive merocyanine-rhodanine dye (NK2761) to monitor neural transmembrane voltage activities. Seven and 8 d old embryonic chick brainstems were sliced into 1400$1600 \mu \mathrm{m}$ thick sections with the glossopharyngeal and vagal nerves attached, and then stained with the dye. Neural voltage-related optical signals were evoked by a positive brief (depolarizing) square current pulse applied to the glossopharyngeal nerve with a microsuction electrode, and then recorded simultaneously from many loci in the objective two-dimensional image plane of a compound microscope. In' addition to the multiple-site optical recording technique, we tried to introduce an optical sectioning method by changing the focal plane of the microscope to obtain three-dimensional information. Thus, we have been able to assess semiquantitatively the three-dimensional profiles of two glossopharyngeal response areas corresponding to the nucleus of the glossopharyngeal nerve (nucleus nervi glossopharyngei) and the nucleus of the tractus solitarius. Furthermore, glutaminergic excitatory postsynaptic potentials were determined within the response area corresponding to the nucleus of the tractus solltarius. In addition, we also compared the glossopharyngeal and vagal response areas and found that the cores of the related nuclei are separated in three dimensions.

[Key words: voltage-sensitive dye, multiple-site optical recording, optical sectioning, embryonic brainstem, glossopharyngeal response, three-dimensional patterns]

In developmental neurobiology/neurophysiology, the understanding of evolution of the spatial patterning of electrical activity in CNSs is a fundamental problem. Because early embryonic central nervous cells and processes are very small and fragile, it is technically very difficult or impossible to impale them

Received Mar. 4, 1994; revised Sept. 26, 1994; accepted Sept. 30, 1994

We are grateful to Larry Cohen and Brian Salzberg for their thoughtful reading of the manuscript and useful comments and suggestions. We also thank Daniel Cohen for his helpful comments in preparing the manuscript. This work was supported by grants from the Ministry of Education, Science and Culture of Japan, and funds from the Mitsubishi Foundation, the Uehara Life Science Memorial Foundation, and Research Aid of the Inoue Foundation for Science.

Correspondence should be addressed to Kohtaro Kamino, Department of Physiology, Tokyo Medical and Dental University School of Medicine, 1-5-45 Yushima, Bunkyo-ku, Tokyo 113, Japan.

Copyright (C) 1995 Society for Neuroscience $0270-6474 / 95 / 152123-18 \$ 05.00 / 0$ with microelectrodes. For this reason, in contrast with morphological investigations, electrophysiological studies of the functional organization of the early embryonic vertebrate CNS have been hampered.

The use of voltage-sensitive dyes and the introduction of multielement photodiode arrays have provided powerful techniques for monitoring the dynamic patterns of neural activity from a variety of invertebrate (Salzberg et al., 1977; Grinvald et al., 1981; London et al., 1987) and vertebrate (Orbach and Cohen, 1983; Salzberg et al., 1983; Grinvald et al., 1984; Orbach et al., 1985; Kauer et al., 1987; Cinelli and Salzberg, 1990, 1992; Fukunishi et al., 1992; Litaudon and Cattarelli, 1992; Taniguchi and Nasu, 1993; Albowiz and Kuhnt, 1993) CNSs. Multiple-site optical recording techniques have facilitated simultaneous re-

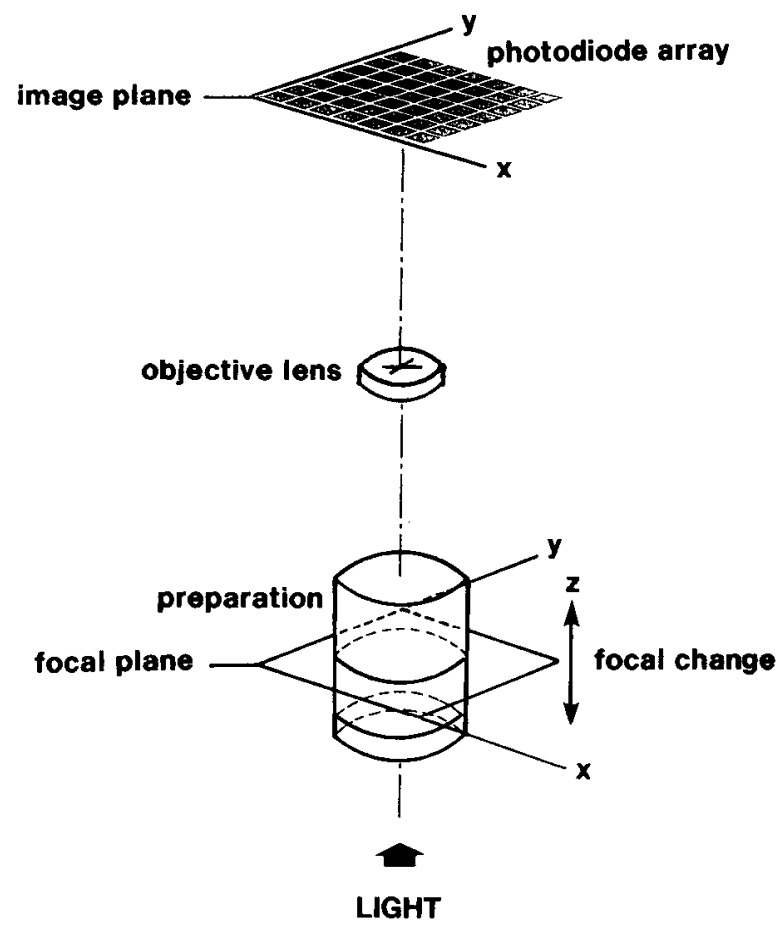

Figure 1. Schematic representation of the optical sectioning method used. The focal plane within the preparation was changed by moving the microscope stage up or down. The recorded signal would be composed of in-focus signal from the focal plane and out-of-focus blurred signals from the rest of the preparation. The optical signals were recorded simultaneously from multiple sites within the image plane using a $12 \times 12$ element photodiode array. See Materials and Methods, and Discussion for additional details. 
Figure 2. Multiple-site optical recordings of neural responses to glossopharyngeal stimulation in intact preparations of a $7 \mathrm{~d}(A)$ and an $8 \mathrm{~d}$

(B) old embryonic chick brainstems. The preparations were stained with a merocyanine-rhodanine dye (NK2761), and the optical signals were evoked by applying a bricf positive square current pulse ( $7 \mu \mathrm{A} / 5 \mathrm{msec}$ for recording $A ; 8$ $\mu \mathrm{A} / 5 \mathrm{msec}$ for recording $B$ ) to the right glossopharyngeal nerve bundles with a microsuction electrode. The evoked optical signals were detected from the ventral side of the brainstems with a $703 \pm 15 \mathrm{~nm}$ interference filter, and in a single sweep. The 127 site simultaneous recordings were made in eight $(A)$ and seven $(B)$ different contiguous areas by sliding the photodiode array over the image of the preparation. Relative positions of the photodiode array with respect to the image of the preparations are illustrated on the lower left corners of $A$ and $B$. The dashed lines correspond to the midline of each preparation. The photodiode array was positioned over a $25 \times$ magnified image of the brainstem, so that each trace represents signals detected by one photodiode from a two-dimensional $56 \times 56$ $\mu \mathrm{m}^{2}$ area of the preparation. The outputs of the individual detectors were divided by the resting level to compensate for the DC background intensity. The direction of the arrows on the right of each recording indicates a decrease in transmitted light (an increase in bound dye absorption) and the lengths of the arrows represent the stated value of the fractional change. The two response areas (area I and area II) are approximately surrounded by two circles: the upper circle indicates area II and the lower circle indicates area I.
A

\section{N. IX /7-day}

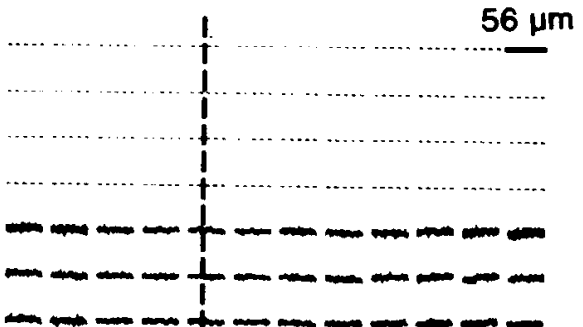

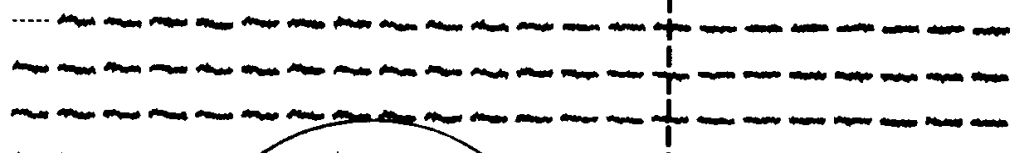

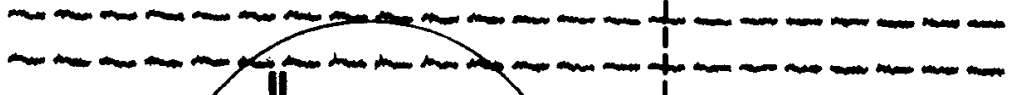

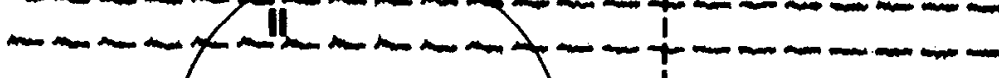

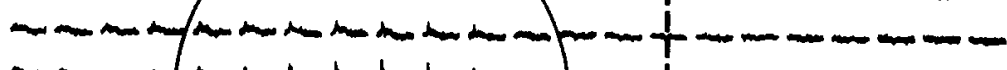

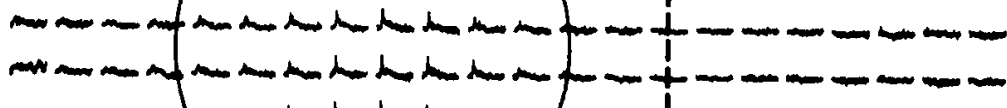

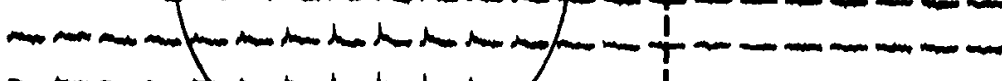

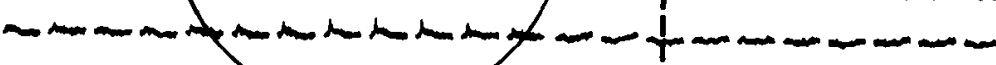

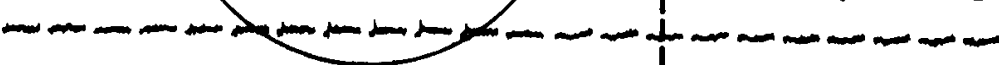
-

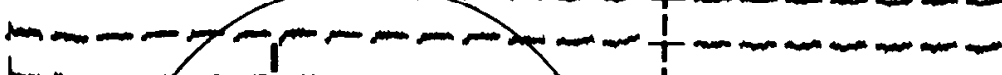

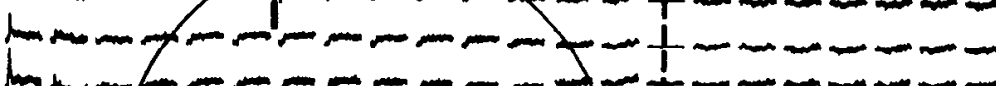

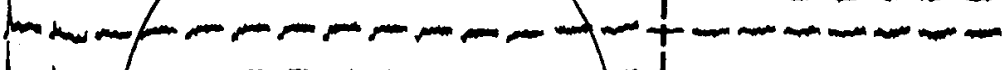

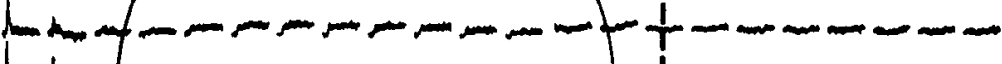

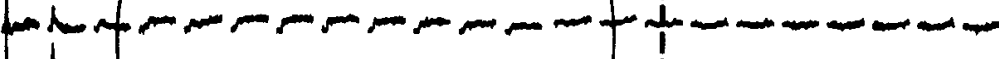

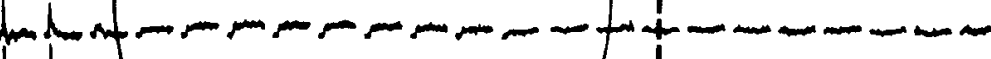

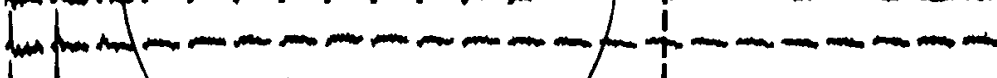

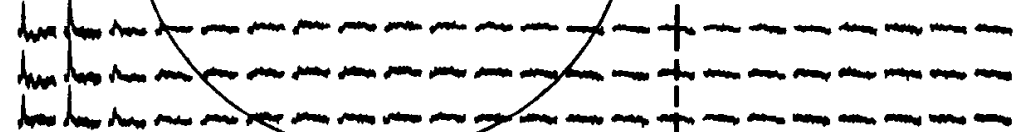

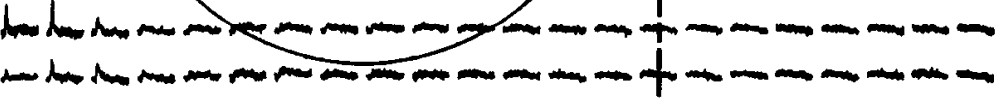

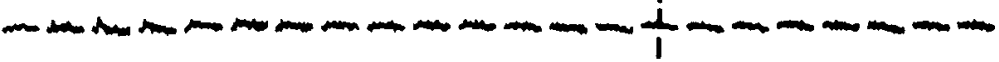
-

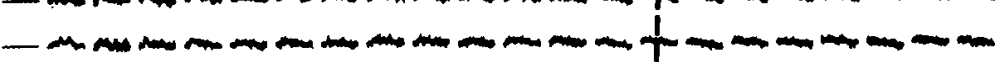

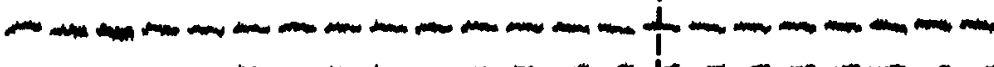

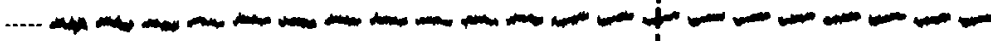

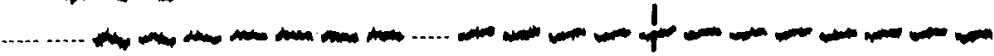
(J835)

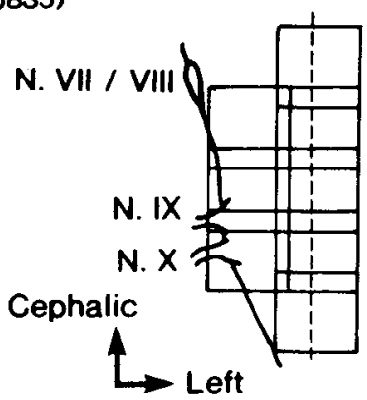

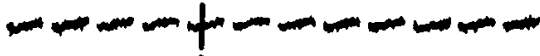

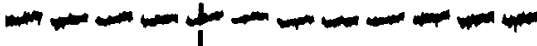
2x10

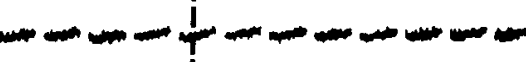


N. IX / 8-day

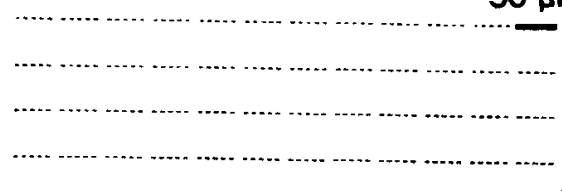

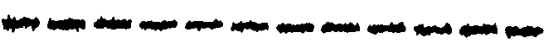

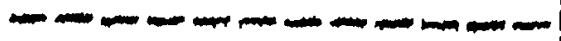

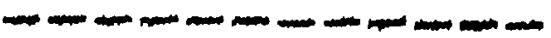

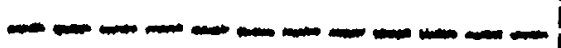

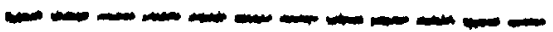

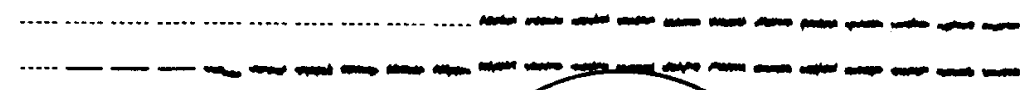

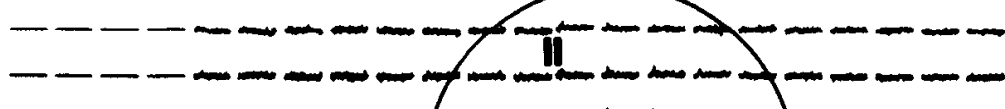
ד

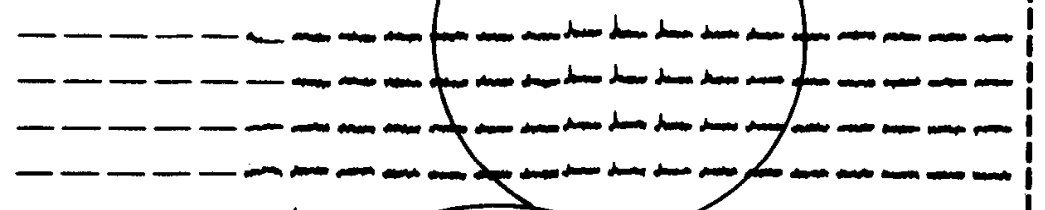

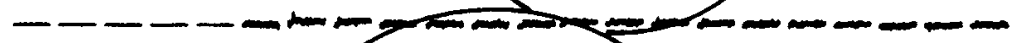

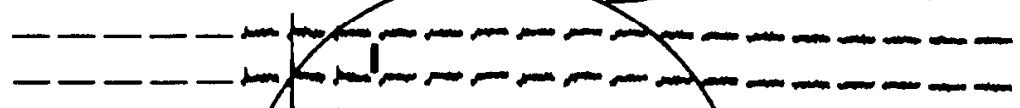

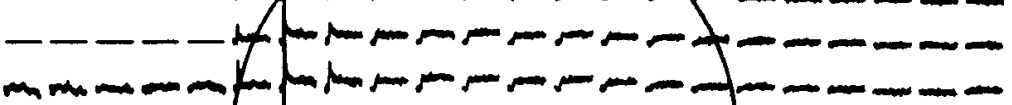

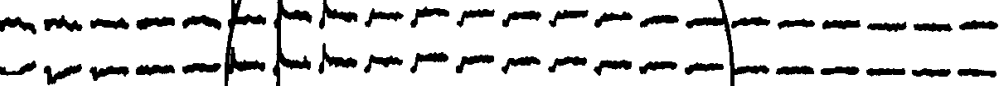

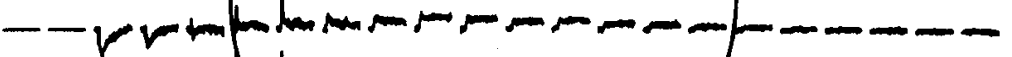
- - -

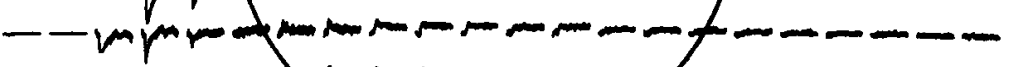

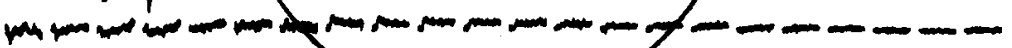
-

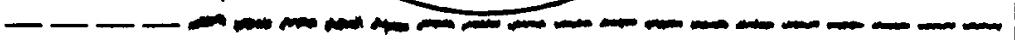

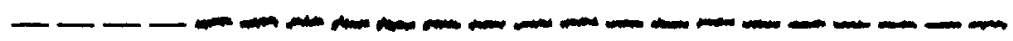

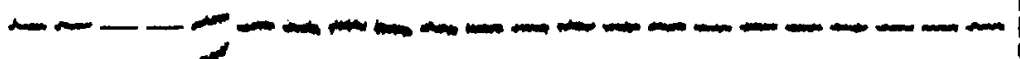

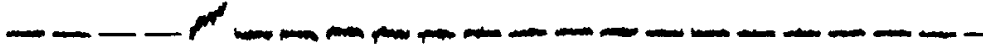

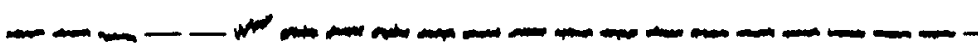

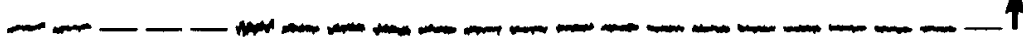

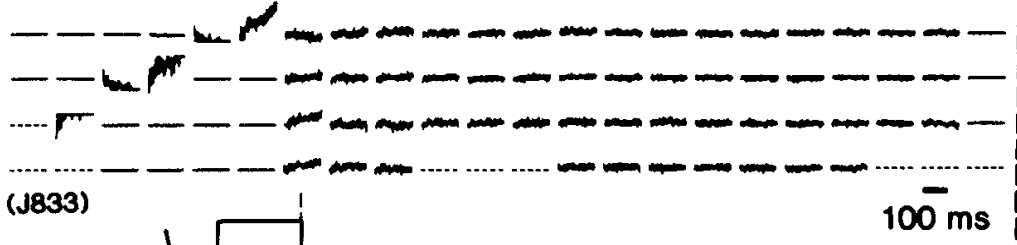

N. VII / VIII

N. IX

Cephalic

N. $x \mp$

1 Left

Figure 2. Continued. 


\section{A}

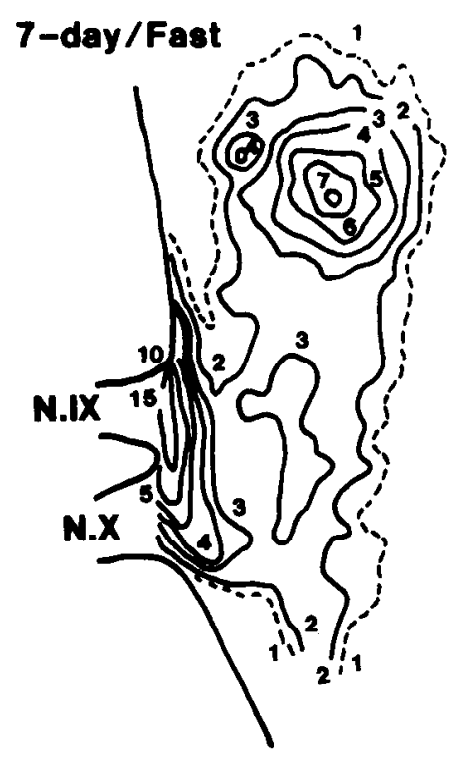

B

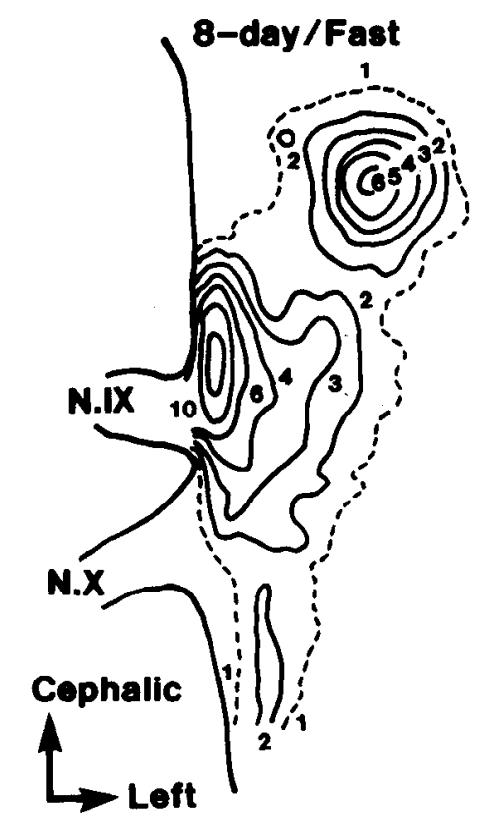

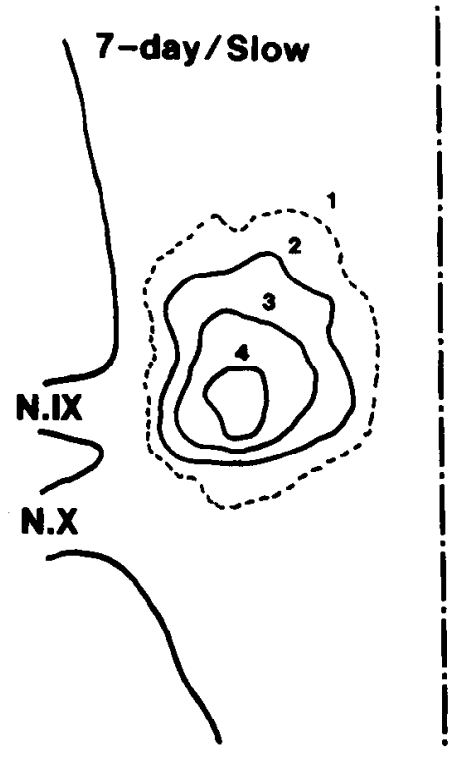

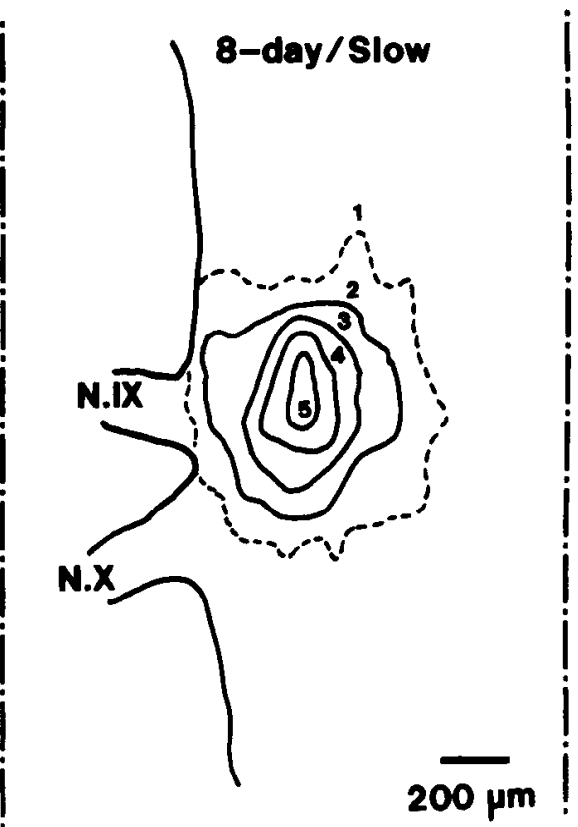

Figure 3. Contour line maps of the size of the evoked optical signals shown in Figure 2. One contour interval represents an increment of $1.0 \times$ $10^{-4}$ in the size of the optical signals; the numerals on the lines indicate the fractional changes multiplied by $10^{4}$. The dashed lines represent the signal size of $1.0 \times 10^{-4}$. Map A is for the 7 $\mathrm{d}$ old embryonic preparation, and map $B$ is for the $8 \mathrm{~d}$ old embryonic preparation. In $A$ and $B$, the maps on the left side are for the fast spike-like signals in each preparation, and the maps on the right side are for the slow signals. cordings of electrical activity from many regions in one preparation (for reviews, see Cohen and Lesher, 1986; Grinvald et al., 1988; Kamino, 1991).

Using such methods, we previously investigated the emergence of electrical activity evoked by vagal stimulation in embryonic brainstems at early developmental stages, and we demonstrated that distinct glutaminergic excitatory postsynaptic potentials are generated in $7 \mathrm{~d}$ old embryonic brainstems (Komuro et al., 1991; Momose-Sato et al., 1991).

Subsequently, in comparison with the vagal response activity, questions have arisen regarding glossopharyngeal response patterns. Preliminary explorations have suggested that glossopharyngeal neural activity is distributed over a relatively extensive area in the early embryonic brainstem, so that measurements in thick-slice preparations are required for optical recording. At the same time, current optical recording tech- niques provide only two-dimensional information concerning neuron positions in the $\mathrm{x}-\mathrm{y}$ plane (object plane of the microscope), with little or no three-dimensional information in the depth axis (vertical axis perpendicular to the object plane of the microscope). This limitation of the optical technique has caused serious problems for assessing the three-dimensional distribution of neural activity in thick preparations. As one way of improving spatial resolution, by analogy with three-dimensional image processing for microscopic analysis (e.g., Agard and Sedat, 1983; Agard, 1984; Erhardt et al., 1985), mathematical deconvolution of results obtained from optical sections recorded at different focal planes has been suggested (for a review, scc Grinvald ct al., 1988).

These optical sectioning methods for exploring the three-dimensional distribution of neural response-related optical signals in CNS preparations should be applicable to our problem. In the 


\section{N. IX/8-day}

\section{$0 /$ surface}

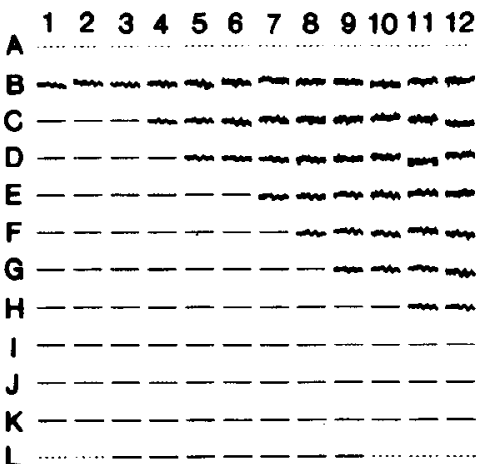

\section{$400 \mu \mathrm{m}$}

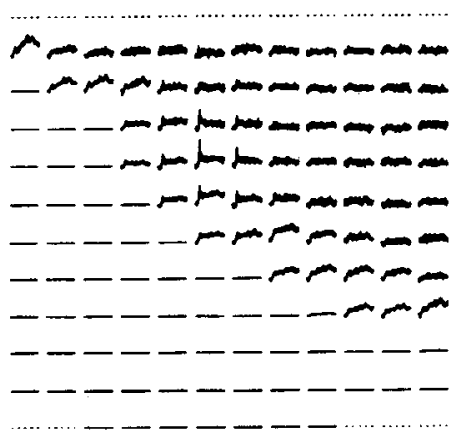

$600 \mu m$

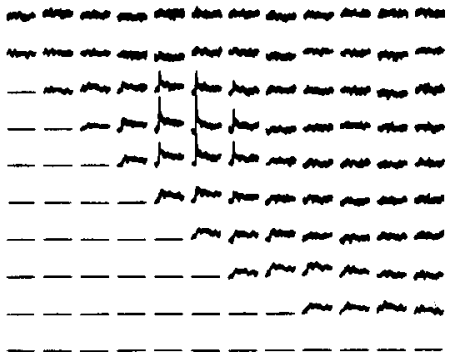

\section{$1000 \mu \mathrm{m}$}

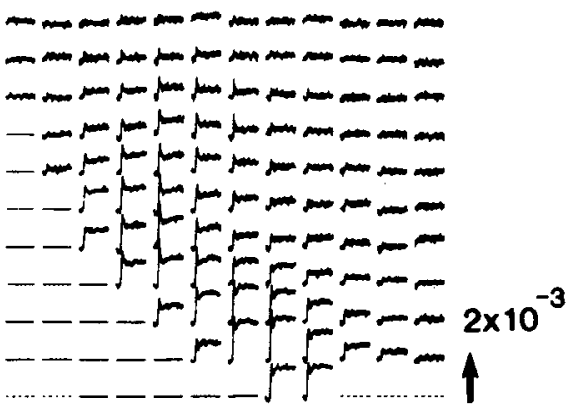

$1200 \mu \mathrm{m}$
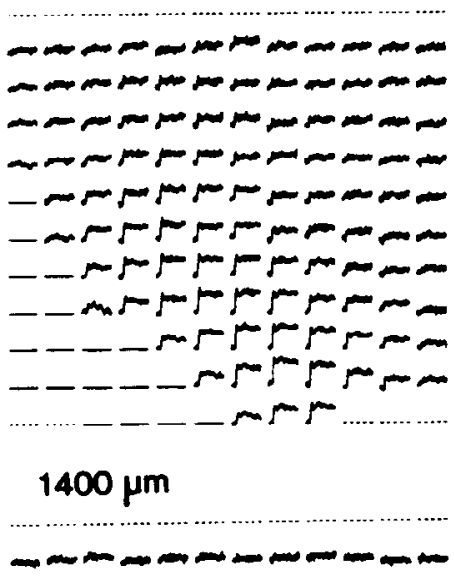

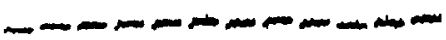

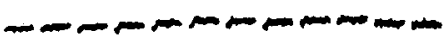

-

-

-

-

- - -

- - - -

- - - - - - -

-

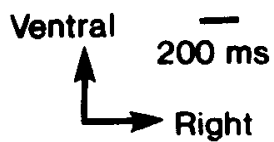

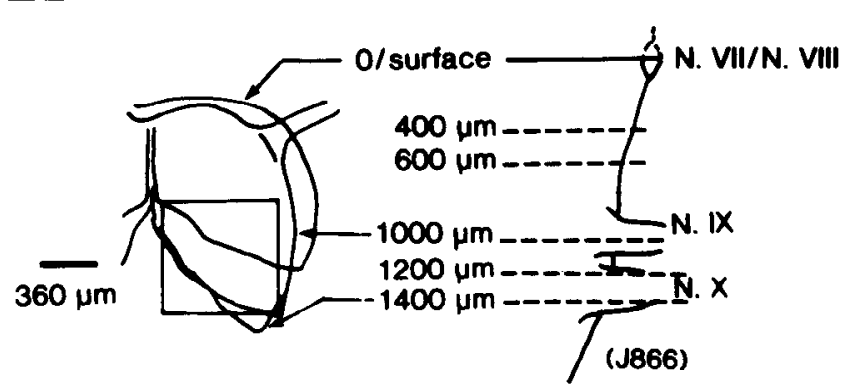

Figure 4. An example of optical multiple-site/sectioning recordings of neural activity evoked by glossopharyngeal stimulation in the thick-slice preparation. The thickness of the slice preparation was about $1600 \mu \mathrm{m}$. A positive square current pulse $(8 \mu \mathrm{A} / 5 \mathrm{msec})$ was applied to the right glossopharyngeal nerve, and the evoked signals were recorded from multiple sites in the $x-y$ plane of the slice preparation at various focal positions. The objective reccived the light transmitted through the preparation in the direction from caudal to cephalic surfaces. The relative position of the photodiode array with respect to the slice at the surface $1000 \mu \mathrm{m}$ depth and $1400 \mu \mathrm{m}$ depth is illustrated at the bottom, and the positions of the focal planes in the cephalic-caudal axis are shown on the lower right. The stated values on each recording indicate the depth from the upper (cephalic) surface of the preparation. present report, we describe the spatial patterning of the glossopharyngeal/vagal response in the early embryonic chick brainstem, as assessed by an empirical/semiquantitative optical sectioning version of classical voltage-sensitive dye recording techniques.

Some of the results presented herein have been communicated in preliminary abstracts (Momose-Sato et al., 1993; Sakai et al., 1993).

\section{Materials and Methods}

The methods used for multiple-site optical recording of electrical activity in embryonic brainstem preparations have been described in detail elsewhere (Komuro et al., 1991; Momose-Sato et al., 1991).

Preparations. In the present experiments, we used intact and slice preparations from the brainstems of chick embryos. Fertilized eggs of White Leghorn chickens were incubated for 7 or $8 \mathrm{~d}$ in a forced-draft incubator (type P-03, Showa Incubator Lab., Urawa, Japan) at a temperature of $37^{\circ} \mathrm{C}$ and $60 \%$ humidity, and were turned once each hour. 
N. IX

Signal-size $\left(\times 10^{-4}\right)$

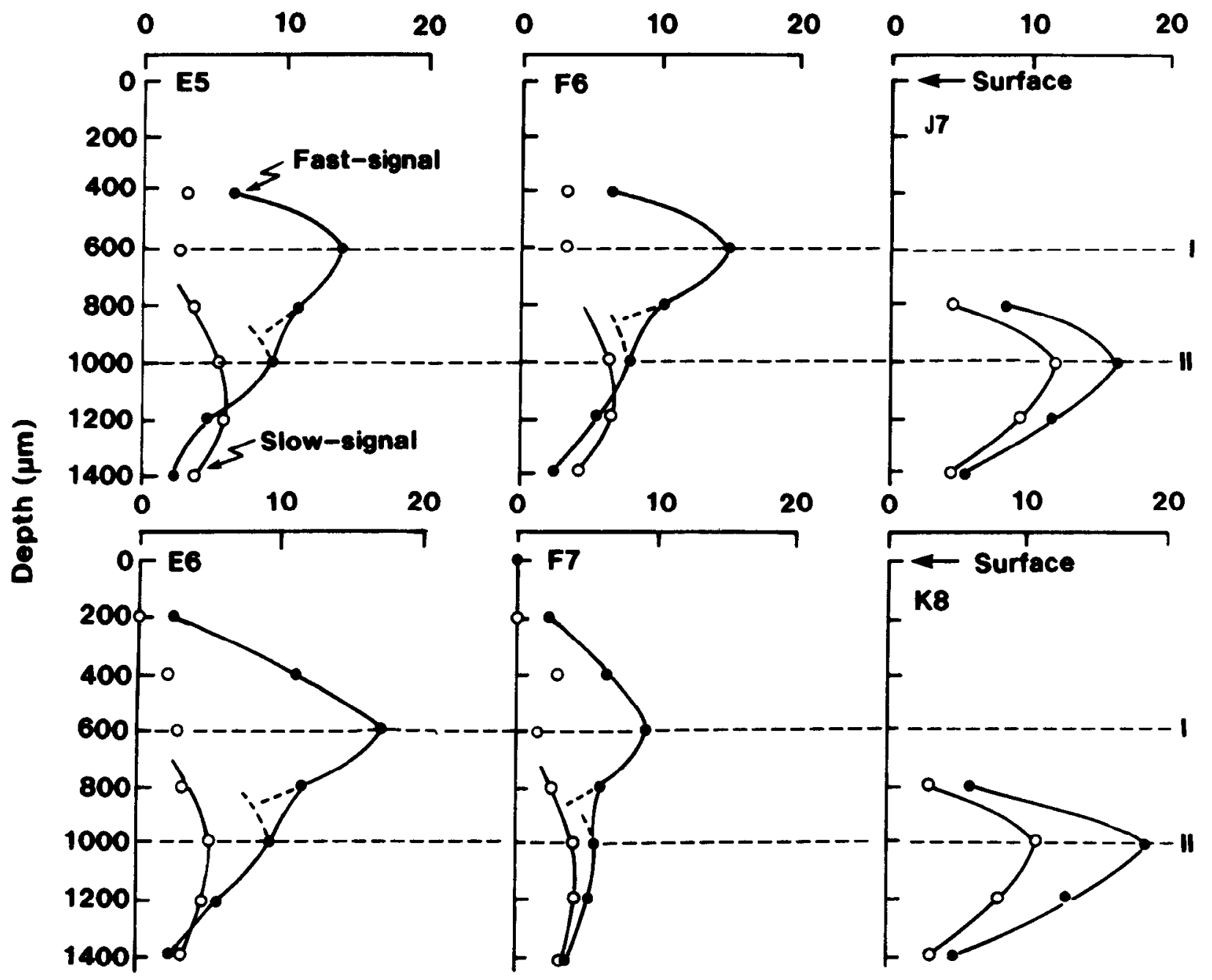

Figure 5. Plots of the size of optical signals against the depth of the plane of focus. The size of the optical signal is expressed as the fractional change. Filled circles are for the fast spike-like signals, and open circles for the slow signals. Data were obtained from the medial region (positions $E 5, E 6, F 6$, and F7) and lateral region (positions $I 7$ and $K 8$ ). Horizontal dashed lines labeled $I$ and $I I$ are placed at the peaks of type I and type II curves for the fast spike-like signals. See Results for additional details.

The brainstems, with glossopharyngeal and vagus nerve fibers attached, were dissected from the embryos. Isolated brainstem preparations were attached to the silicone (KE 106LTV; Shin-etsu Chemical Co., Tokyo, Japan) bottom of a simple chamber by pinning it with tungsten wires. Preparations were kept in a bathing solution with the following composition (in $\mathrm{mM}$ ): $\mathrm{NaCl}, 138 ; \mathrm{KCl}, 5.4 ; \mathrm{CaCl}_{2}, 1.8 ; \mathrm{MgCl}_{2}, 0.5$; glucose, 10; and Tris- $\mathrm{HCl}$ buffer ( $\mathrm{pH} \mathrm{7.2),10.} \mathrm{The} \mathrm{solution} \mathrm{was} \mathrm{equilibrated}$ with oxygen. The pia mater attached to the brainstem was carefully removed in the bathing solution under a dissecting microscope. The slice was then prepared, with the right and/or left glossopharyngeal and vagus nerve fibers attached, by sectioning the embryonic brainstem transverscly. The thickness of the slice was $1400-1600 \mu \mathrm{m}$.

Dye staining. Isolated preparations were stained by incubation (for 20-30 min for the intact preparations, or for approximately $30 \mathrm{~min}$ for the slice preparations) in a Ringer's solution containing $0.2 \mathrm{mg} / \mathrm{ml}$ of the voltage-sensitive merocyanine-rhodanine dye NK2761 (Nippon Kankoh Shikiso Kenkyusho, Okayama, Japan; Kamino et al., 1981; Salzberg et al., 1983; Kamino et al., 1989a; Kamino, 1991). Excess (unbound) dye was washed away with dye-free Ringer's solution before recording.

Electrical stimulation. For preparations in which the glossopharyngeal or vagus nerve was stimulated, the cut end of the nerve was drawn into a microsuction electrode fabricated from TERUMO-hematocrit tubing (VC-H075P; TERUMO Co., Tokyo, Japan), which had been hand- pulled to a fine tip (internal diameter, about $50 \mu \mathrm{m}$ for the glossopharyngeal nerve and $100 \mu \mathrm{m}$ for the vagus nerve) over a low-temperature flame.

Optical recording. Light from a $300 \mathrm{~W}$ tungsten-halogen lamp (type JC-24V/300W, Kondo Sylvania Ltd., Tokyo, Japan) was collimated, rendered quasimonochromatic with a heat filter (32.5B-76, Olympus Optical Co., Tokyo, Japan) and an interference filter with a transmission maximum at $703 \pm 15 \mathrm{~nm}$ (Asahi Spectra Co., Tokyo, Japan), and focused on the preparation by means of a bright field condenser with a numerical aperture (NA) matched to that of the microscope objective (S plan Apo, 10×, 0.4 NA). The objective and photographic eyepiece $(2.5 \times)$ projected a real image of the preparation onto a $12 \times 12$ element silicon photodiode matrix array (MD-144-4PV; Centronic Ltd., Croydon, UK) mounted on an Olympus Vanox microscope (type AHB-L-1, Olympus Optical Co., Tokyo, Japan) at a magnification of $25 \times$. Each pixel (element) of the array detected light transmitted by a square region $\left(56 \times 56 \mu \mathrm{m}^{2}\right)$ of the preparation. The output of each detector in the diode array was passed to an amplifier via a current-to-voltage converter. The amplified outputs from 127 elements of the detector were first recorded simultaneously on a 128-channel recording system (RP890 series, NF Electronic Instruments, Yokohama, Japan), and then passed to a computer (LSI-11/73 system, Digital Equipment Co. Tewkshury, MA). The 128 channel data recording system was composed of a main processor (RP-891), eight I/O processors (RP-893), a 
N.IX / 7-day

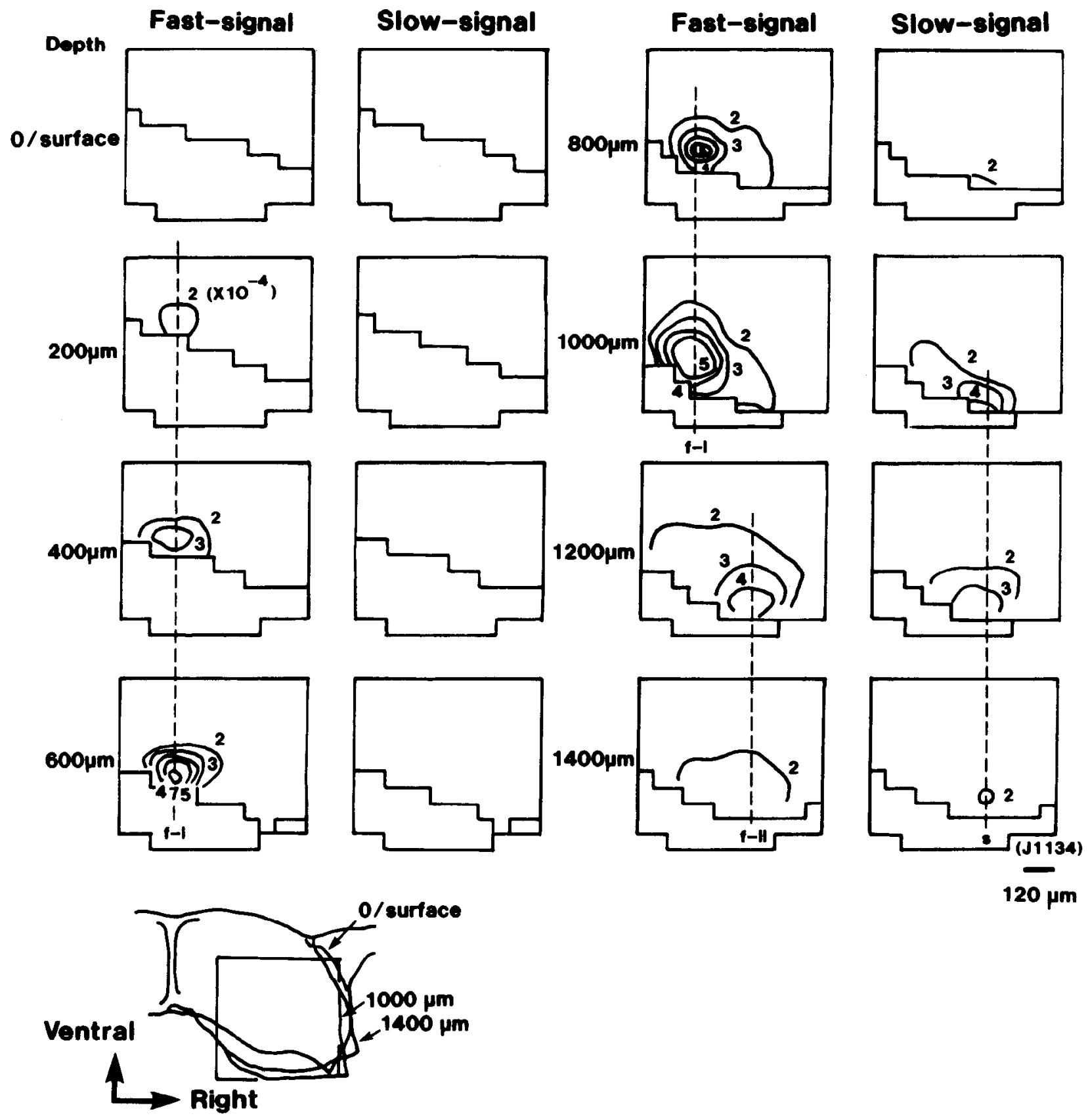

Figure 6. Contour line maps of the size of the optical signals detected at various depths of focus. Data in $A$ were obtained from a $7 \mathrm{~d}$ old embryonic brainstem slice preparation, and data in $B$ were obtained from the preparation shown in Figure 4 . In $A$, the relative positions of the recorded areas of the focal planes (surface, $1000 \mu \mathrm{m}$ and $1400 \mu \mathrm{m}$ ) are illustrated on the lower left corner; the relative positions for $B$ were shown in Figure 4. Vertical dashed lines labeled $f-I$ are placed at the positions of the peak-size area of the fast spike-like signals in the medial region; the dashed lines labeled $f-I I$ are placed at the position of the peak-size area of the fast spike-like signals in the lateral region; the dashed lines labeled $s$ are placed at the positions of the peak-size area of the slow signals.

64K word wave-memory (RP-892) and a videotape recorder. The program for the computer was written in the assembly language (Macro11) called from FORTRAN, under the RT-11 operating system (version 5.0). Optical recording was carried out in a still chamber without continuous perfusion with Ringer's solution, at room temperature, $26-28^{\circ} \mathrm{C}$. The incident light was turned off except during the measuring period. Under these conditions, in 7 and $8 \mathrm{~d}$ old embryonic brainstem preparations, the evoked optical signals could often be detected continuously for 60-150 min. Optical sectioning recordings were carried out by changing the focal plane within the preparation by moving the stage of the microscope, as shown schematically in Figure 1.

\section{Results}

Recordings from intact preparations

Our first experiments were carried out on intact brainstem preparations. Figure 2 illustrates two examples of multiple-site optical recordings of neural activity from intact preparations of a $7 \mathrm{~d}$ and an $8 \mathrm{~d}$ old embryonic chick brainstem stained with NK2761. Neural activity was evoked by a brief square current pulse applied to the right glossopharyngeal nerve with a microsuction electrode. (In the same $8 \mathrm{~d}$ old preparation, the right 
N.IX / 8-day

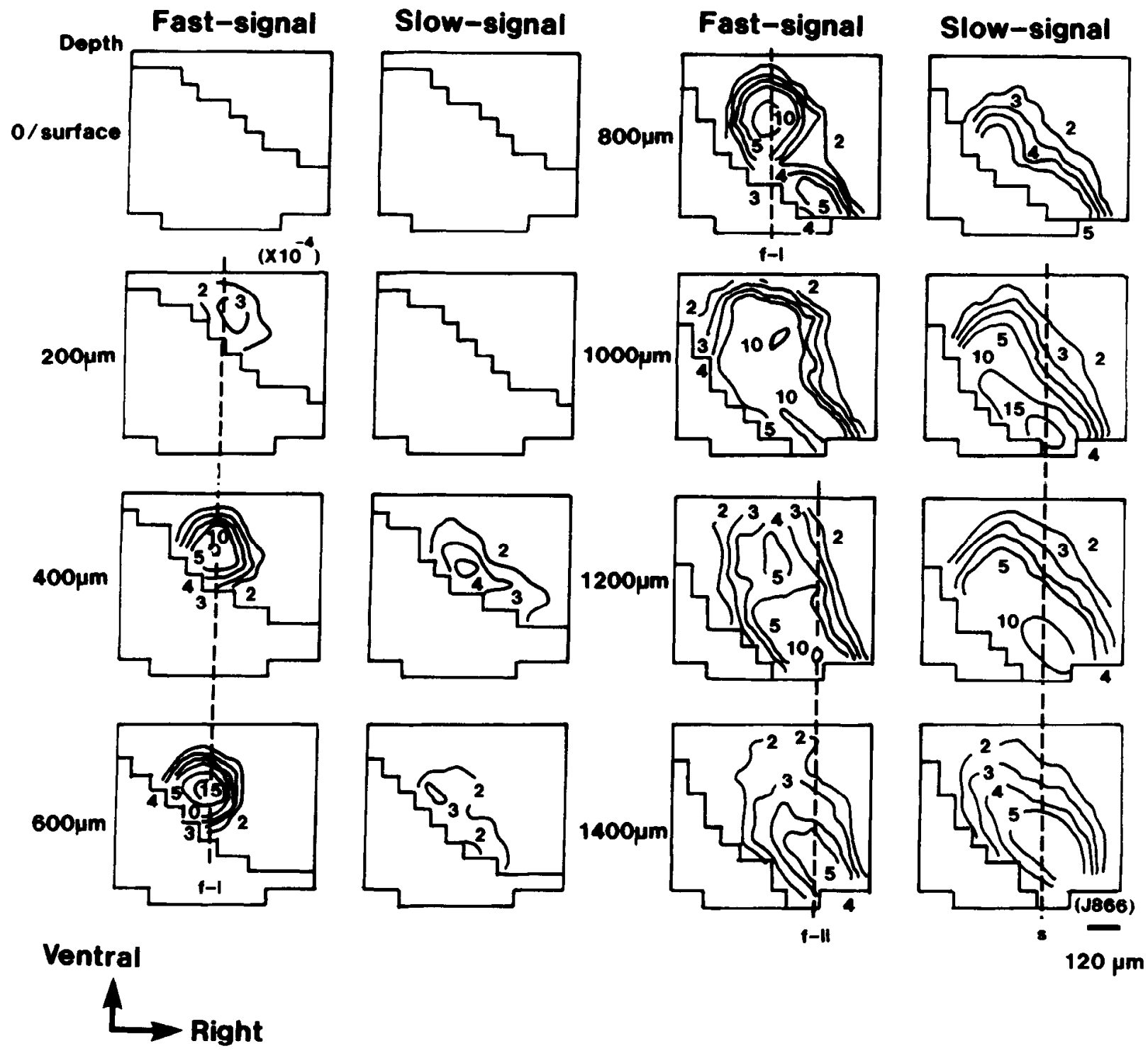

Figure 6. Continued.

vagus nerve was also stimulated. The recording is shown in Figure 9, and is compared with the glossopharyngeal recordings). The thicknesses (light-path length from the dorsal surface to ventral surface) of these intact embryonic brainstem preparations were $700-1000 \mu \mathrm{m}$, and they were translucent. Thus, we could detect neural voltage responses as changes in transmitted light intensity. The evoked optical signals were recorded simultaneously from 127 contiguous regions of the preparation using a $12 \times 12$ element photodiode array. Simultaneous 127 site optical recordings were made in seven or eight areas of the preparation by moving the photodiode array on the image of the preparation, and the recordings displayed in Figure 2 were reconstructed by combining the individual simultaneous recordings.

When a stimulating current $(7.0 \mu \mathrm{A} / 5.0 \mathrm{mscc}$ for the $7 \mathrm{~d}$ old preparation and $8.0 \mu \mathrm{A} / 5.0 \mathrm{msec}$ for the $8 \mathrm{~d}$ old preparation) was applied to the right glossopharyngeal nerve, the evoked optical signals were concentrated in two limited areas: one located near the level corresponding to the root of the glossopharyngeal/ vagus nerve (area I), and the other located cephalic to the level of the glossopharyngeal nerve (area II). In both the $7 \mathrm{~d}$ and $8 \mathrm{~d}$ old preparations, only fast spike-like signals were significantly recorded from area II, while signals consisting of two components, viz., a spike-like signal and a delayed long-lasting slow signal, were recorded from area $I$ in all preparations tested $(n \cong$ 50) (see also Fig. 8). Consistent with previous papers on the vagal responses (Komuro et al., 1991; Momose-Sato et al., 1991), we assumed that the fast component of the optical signal corresponds to the action potential and the slow component to the postsynaptic potential. Some physiological characteristics of the slow signals are described below.

The sizes of the glossopharyngeal signals were relatively small, in comparison with vagal responses (see Fig. 9). And, with vagal stimulation, no response area corresponding to "area II" was found.

Mapping. In order to evaluate the response area quantitatively, 


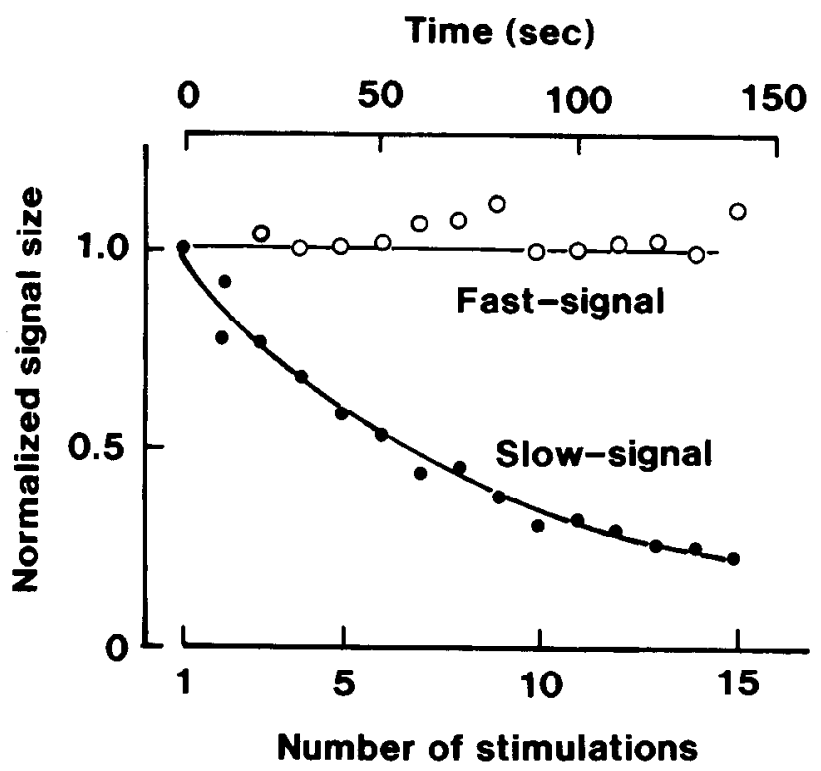

Figure 7. Decreasing slow signal size with continuous stimulation in an $8 \mathrm{~d}$ old embryonic brain stem slice preparation. Square current pulses of $8.0 \mu \mathrm{A} / 5.0 \mathrm{msec}$ and $0.1 \mathrm{~Hz}$ were applied to the right glossopharyngeal nerve. The relative amplitudes of the fast (open circles) and slow (solid circles) signals are plotted against the stimulation number. The upper abscissa indicates the time in seconds.

we measured the sizes (fractional changes $=$ the changes in transmitted light intensity divided by the DC-background intensity) of the fast spike-like and slow signals, and we constructed contour line maps of the signals. In Figure 3, the maps constructed using the data obtained from the recordings shown in Figure 2 are illustrated. In these contour line maps, area I appears to correspond to the slow signal response area, and area II seems to correspond to the fast spike-like signal response area. In both areas, the distribution of the signal sizes appeared to form a layered pattern. The peak-size area of the slow signal was located at the level of the root of the glossopharyngeal nerve (area I), and that of the fast spike-like signal was found in the cephalic region about $500 \mu \mathrm{m}$ from the level of the glossopharyngeal nerve (area II). Small fast spike-like signals were also detected in area $I$.

\section{Optical sectioning recording from thick-slice preparations}

It is difficult to obtain three-dimensional information from the experiments on intact preparations. To circumvent this limitation, slice preparations would be useful. However, as observed from the intact recordings and the contour line maps shown in Figures 2 and 3, the glossopharyngeal response area was distributed widely from the level of the glossopharyngeal nerve to the cephalic region. Thus, a thin transverse slice preparation at the level of the glossopharyngeal nerve would not provide adequate information concerning the $\mathrm{z}$-axis (caudal-cephalic)/ $\mathrm{x}-\mathrm{y}$ plane (object plane of the microscope: transverse section plane) distribution of the response signals. As a possible means of evaluating the $z$-axis/ $\mathrm{x}-\mathrm{y}$ plane distribution of the response, an optical sectioning recording might prove very useful. Thus, we have tried to apply the optical sectioning method to thick-slice preparations.

The brainstem was sliced into a block having the glossopharyngeal nerve attached and containing two glossopharyngeal response areas ("area I" and "area II") identified in Figures 2 and 3. The thickness from cephalic surface to caudal surface of each block was $1400-1600 \mu \mathrm{m}$. After the block was stained in a bathing solution containing NK2761 for $30 \mathrm{~min}$, it was placed on the bottom of a chamber with the cephalic side facing upward, and the chamber was placed on the microscope stage.

Figure 4 shows a series of the optical multiple-site/sectioning recording of glossopharyngeal response activity in a thick-slice preparation from an $8 \mathrm{~d}$ old embryonic brainstem. Changing the focal plane of the objective by moving the microscope stage along the z-axis (caudal-cephalic), we recorded the evoked response signals simultaneously from multiple loci at eight focal positions: 0 (cephalic surface), 200, 400,600,800, 1000, 1200 , and $1400 \mu \mathrm{m}$ from the surface of the preparation. A position of $1000 \mu \mathrm{m}$ corresponded to the level of the root of the glossopharyngeal nerve, and a depth of $1400 \mu \mathrm{m}$ corresponded to the level of the vagus nerve.

In the recording shown in Figure 4, when the microscope objective was focused just at the surface plane, no evoked signals were detected. Within the depth range of $200-1400 \mu \mathrm{m}$, evoked optical signals were detected, and these were concentrated in the dorsal region of each focal plane. Due to the threedimensional morpholngical structure of the preparation, some photodiode elements were located outside the boundary of the preparation, so that there were no signals in the corresponding positions. These detectors are represented as flat lines. The signal size and response area varied with the depth of the focal plane.

In the present optical sectioning recording, it is important to determine the depth to which the dye diffuses into the thickslice preparation (block). Therefore, using the thinner slice preparations made from the stained thick-slice preparation, we examined the dye staining in deeper regions, with microscopic observations and measurements of DC-background transmitted light intensity, confirming that the dye diffused throughout the depth of the thick preparation. The immature cellular-interstitial structure of the early embryonic brainstem may allow the dye to diffuse readily from the surface to the interior regions.

In Figure 5, the sizes of the recorded fast and slow signals are plotted against the depth of the focal plane of the preparation. The data were obtained from the recording shown in Figure 4 . The signal-size is expressed as a fractional change and the depth is expressed as distance from the surface of the preparation. From these graphical representations, one expects to obtain the global features of the z-axis/x-y plane distributions of the fast and slow signals. Figure 5 shows two kinds of curves for the fast spike-like signals. One (type I) is a roughly parabolic curve having its maximum value near a depth of $600 \mu \mathrm{m}$, and the other (type II) is a curve having its maximum value near $1000 \mu \mathrm{m}$. From the medial side (compare positions E5, E6, F6, and F7), both type I and type II profiles were obtained, and from the lateral side (compare positions $\mathrm{J} 7$ and K8), only type II was obtained. For the slow signal, only one kind of curve was found, having its maximum value near a depth of $1000 \mu \mathrm{m}$, and this curve paralleled the type II curve for the fast spike-like signal. The horizontal dashed lines with labels I and II are placed at the peak depths for type I and type II of the fast signal curves.

The graphical characteristics of the signal size versus depth relationship suggested an interpretation of the z-axis distribution of the glossopharyngeal response signals. The type I fast signal curve appeared to correspond to area II identified in Figures 2 and 3, and the type II curve for the fast spike-like signal and 

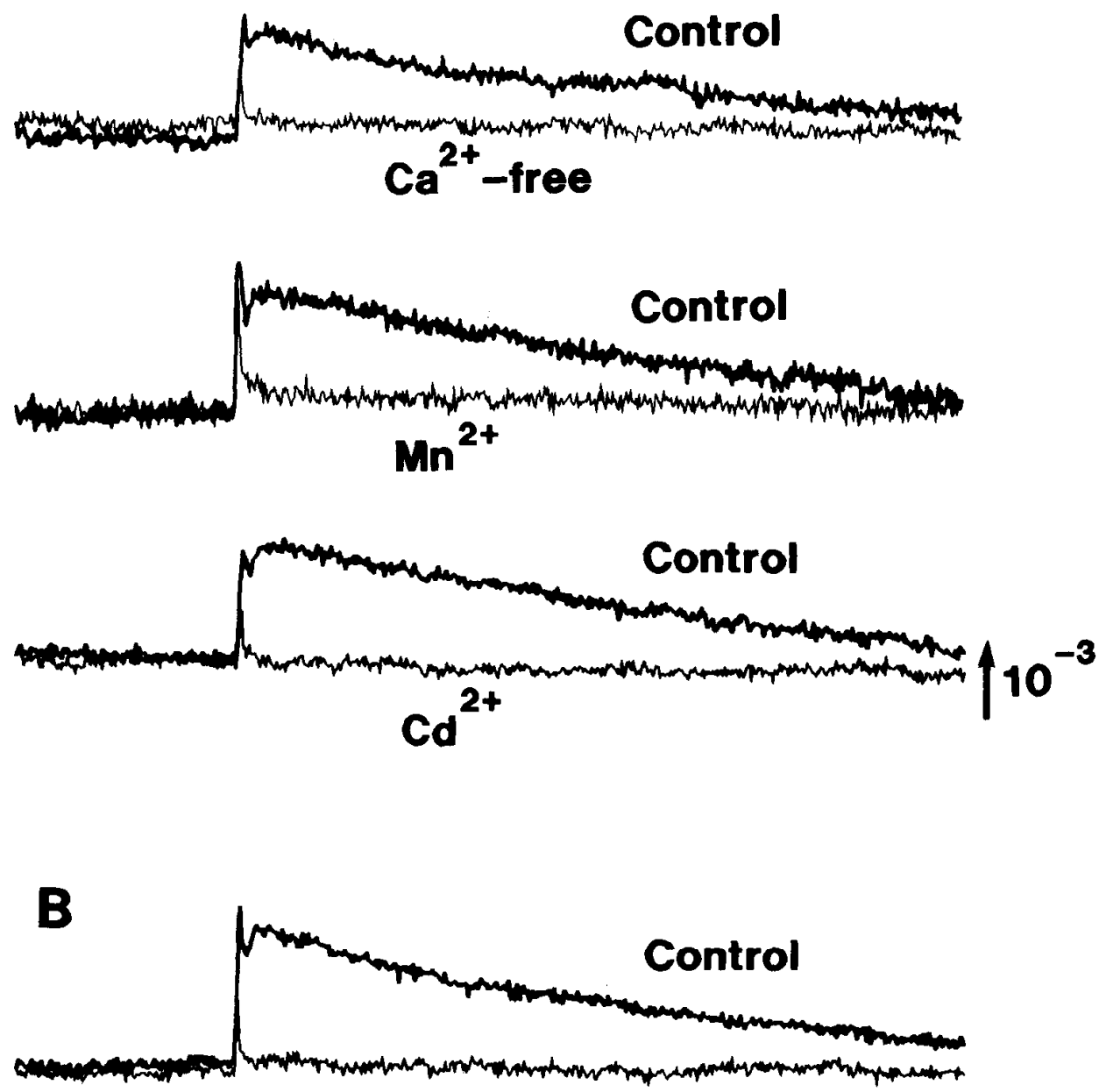

Kynurenic acid

Figure 8. A, Effects of $\mathrm{Ca}^{2+}$-free bathing solution, $\mathrm{Mn}^{2+}(2 \mathrm{mM})$-containing solution and $\mathrm{Cd}^{2+}(1 \mathrm{mM})$-containing solution on the evoked optical signals. Thicker traces were controls, and thinner traces were obtained in the $\mathrm{Ca}^{2+}$-free bathing solution (first panel), the $\mathbf{M n}^{21}$-containing solution (second panel) and the $\mathrm{Cd}^{2+}$-containing solution (third panel). $B$, Effects of kynurenic acid (1.2 mM), 2-APV (200 $\mu \mathrm{M})$, and CNQX ( $5 \mu \mathrm{M})$ on the evoked optical signals. Thicker traces were controls, and thinner traces were obtained in kynurenic acid containing bathing solution (first panel), 2-APV containing solution (second panel), and CNQX-containing solution (third panel).

the curve for the slow signal seemed to correspond to area I determined in the intact recording.

Mapping. Using these data, we have constructed contour line maps of the $x-y$ plane distribution of the sizes of the fast spikelike and slow signals detected in each focal plane. Two examples
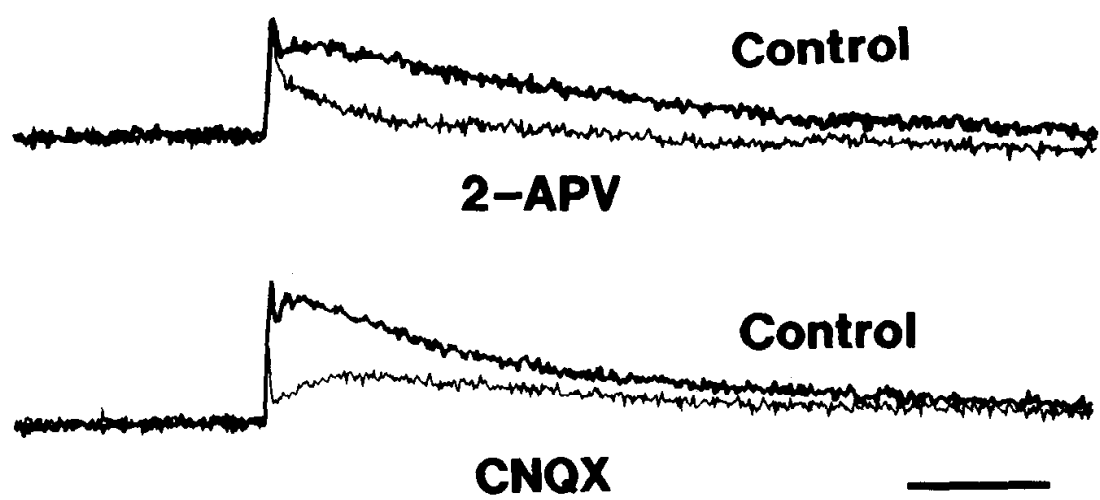

$10^{-3}$

\section{$200 \mathrm{~ms}$}

obtained from the $7 \mathrm{~d}$ and $8 \mathrm{~d}$ old preparations are illustrated in Figure 6 . The distribution of the signal sizes indicated a multiple-layered pattern. In addition, the two distribution patterns corresponding to the type I and type II curves in the fast spike-like signals are clearly shown, and the differences for the peak-size 


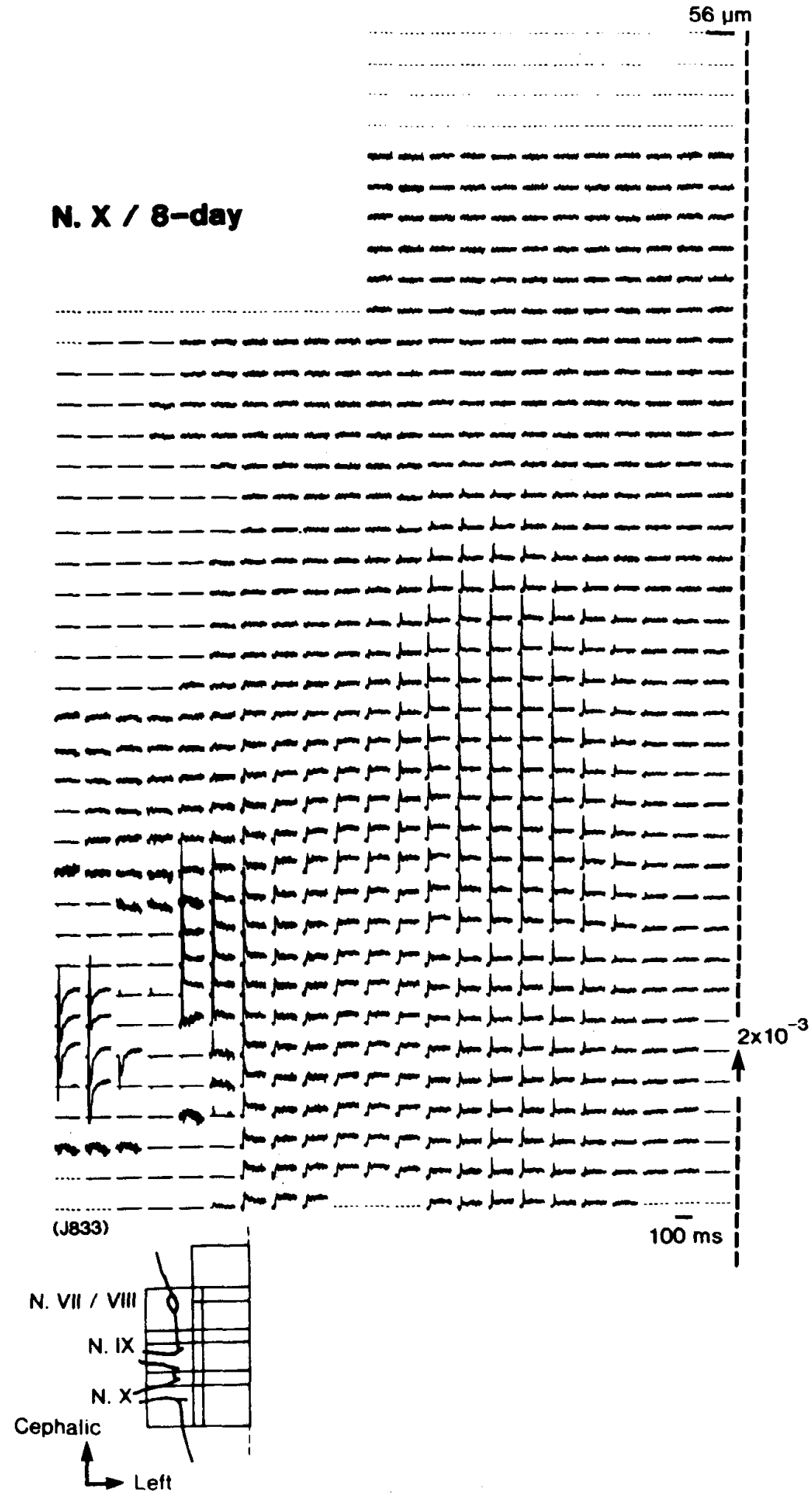

Figure 9. Multiple-site optical recording of the vagal response in the intact $8 \mathrm{~d}$ preparation also used in the experiment shown in Figure $2 B$. A positive square current pulse $(8 \mu \mathrm{A} / 5$ msec) was applied to the right vagus nerve, and simultaneous 127 -site optical recordings were carried out in seven contiguous areas. The dashed line corresponds to the midline of the preparation. The recordings were made in a single sweep. Other experimental conditions and indications are the same as those in Figure $2 B$. areas between the fast spike-like and slow signals were identified. In the $x-y$ plane, the peak-size area of the type I-fast spikelike signal was positioned within the dorsal/medial region, and those of the type II-fast spike-like signal and the slow signal were localized to the dorsal/lateral region. From the maps shown in Figure 6, we obtained the evidence that, although the distribution pattern of the signal size was basically similar between the $7 \mathrm{~d}$ and $8 \mathrm{~d}$ old preparations, the size of the slow signals in the $8 \mathrm{~d}$ old preparation was larger than that in the $7 \mathrm{~d}$ old preparation. 
N. X/8-day

\section{$0 /$ surface}

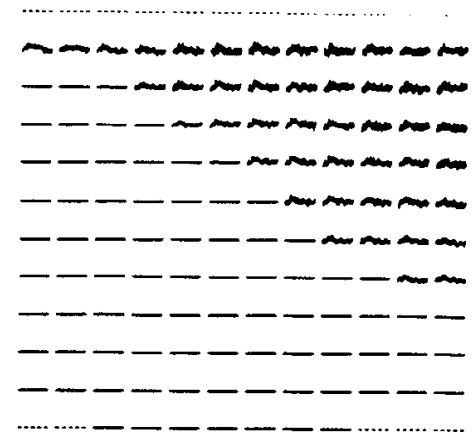

$400 \mu \mathrm{m}$

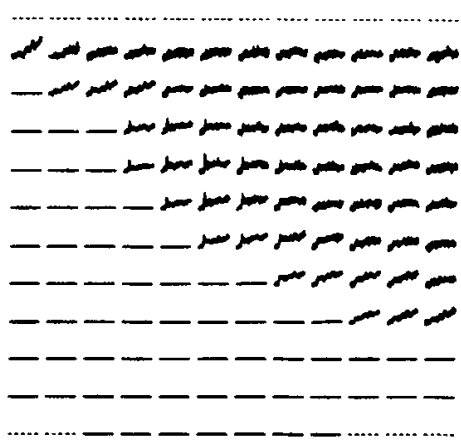

$600 \mu \mathrm{m}$

Figure 10. Optical sectioning recordings of the vagal responses in an $8 \mathrm{~d}$ thick-slice preparation. A positive square current pulse $(8 \mu \mathrm{A} / 5 \mathrm{msec})$ was applied to the right vagus nerve, and the evoked optical signals were detected at various focal planes, using a $12 \times 12$ element photodiode array. The preparation used in this cxpcriment was the same as that shown in Figure 4 Other experimental conditions and indications are also the same as in Figure 4 .

\section{$1000 \mu \mathrm{m}$}
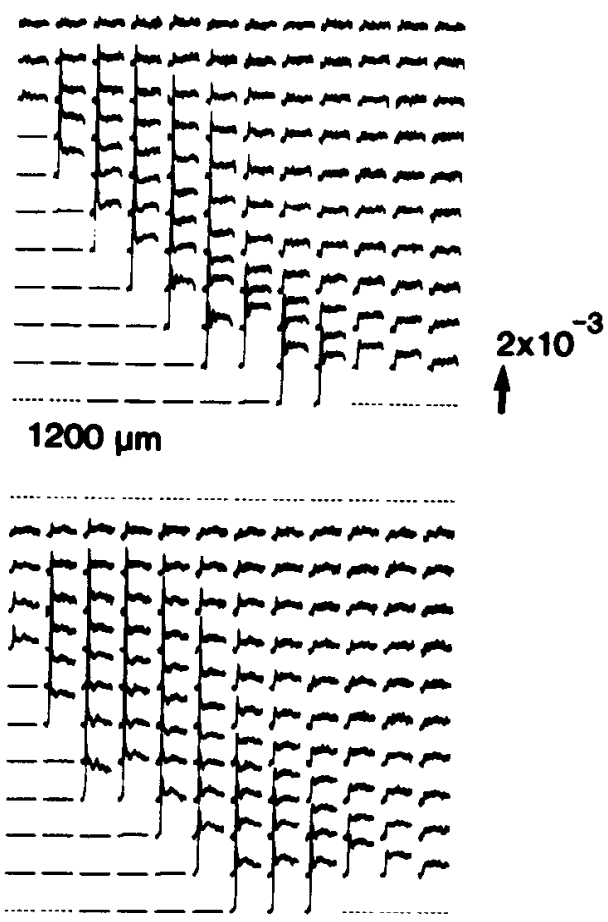

$1400 \mu \mathrm{m}$

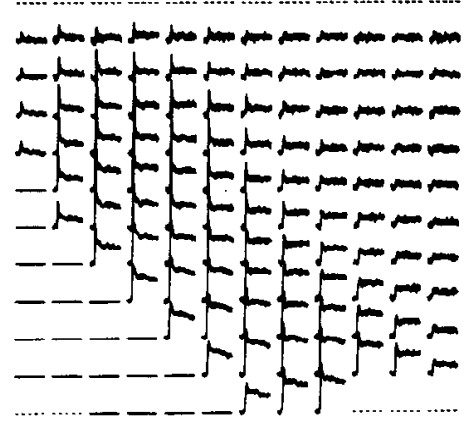

(J806)
The synaptic properties of the slow signal

In the same manner that we have previously measured the vagus nerve response (Komuro et al., 1991), we examined the synaptic properties of the slow signal evoked by the glossopharyngeal stimulus in the present study. The size of the slow signal gradually decreased with continuous stimulations $(0.1 \mathrm{~Hz})$, although the size of the fast spike-like signal was nearly constant (Fig. 7). It is likely that this decline reflects synaptic fatigue and, if so, it argues that the slow signal is intimately related to postsynaptic potential. Also, the slow signals were reduced or eliminated by lowering the external $\mathrm{Ca}^{2+}$ concentration in the bathing solution, and they were eliminated by the addition of $\mathrm{Mn}^{2+}$ or $\mathrm{Cd}^{2+}$ to the bathing solution (Fig. $8 A$ ). Furthermore, the slow signals were blocked by kynurenic acid, and their initial phase was reduced by CNQX (6-cyano-7-nitroquinoxaline-2,3-dione) and the later phase was reduced by 2-APV (DL-2-amino-5-phosphonovaleric acid) (Fig. $8 B$ ). To examine the possibility that other chemical transmitters (such as adrenaline, ACh, GABA, and glycine) play significant roles in the synaptic physiology of the embryonic brainstem, we examined the effects of several other blockers [phentolamine $(20 \mu \mathrm{M})$, propranolol $(10 \mu \mathrm{M})$, $d$-tubocurarine $(190 \mu \mathrm{M})$, atropine $(10 \mu \mathrm{M})$, picrotoxin $(100 \mu \mathrm{M})$, and strychnine $(10 \mu \mathrm{M})]$. We did not find significant effects of any of these blockers on the slow signals. Thus, it seems less likely that other transmitters are active in generating these slow signals. When TTX $(20 \mu \mathrm{M})$ was applied, the fast spike-like signals were eliminated together with the slow signals (data not shown). From these physiological and pharmacological charac- 
N. $X$

Signal-size $\left(\times 10^{-4}\right)$

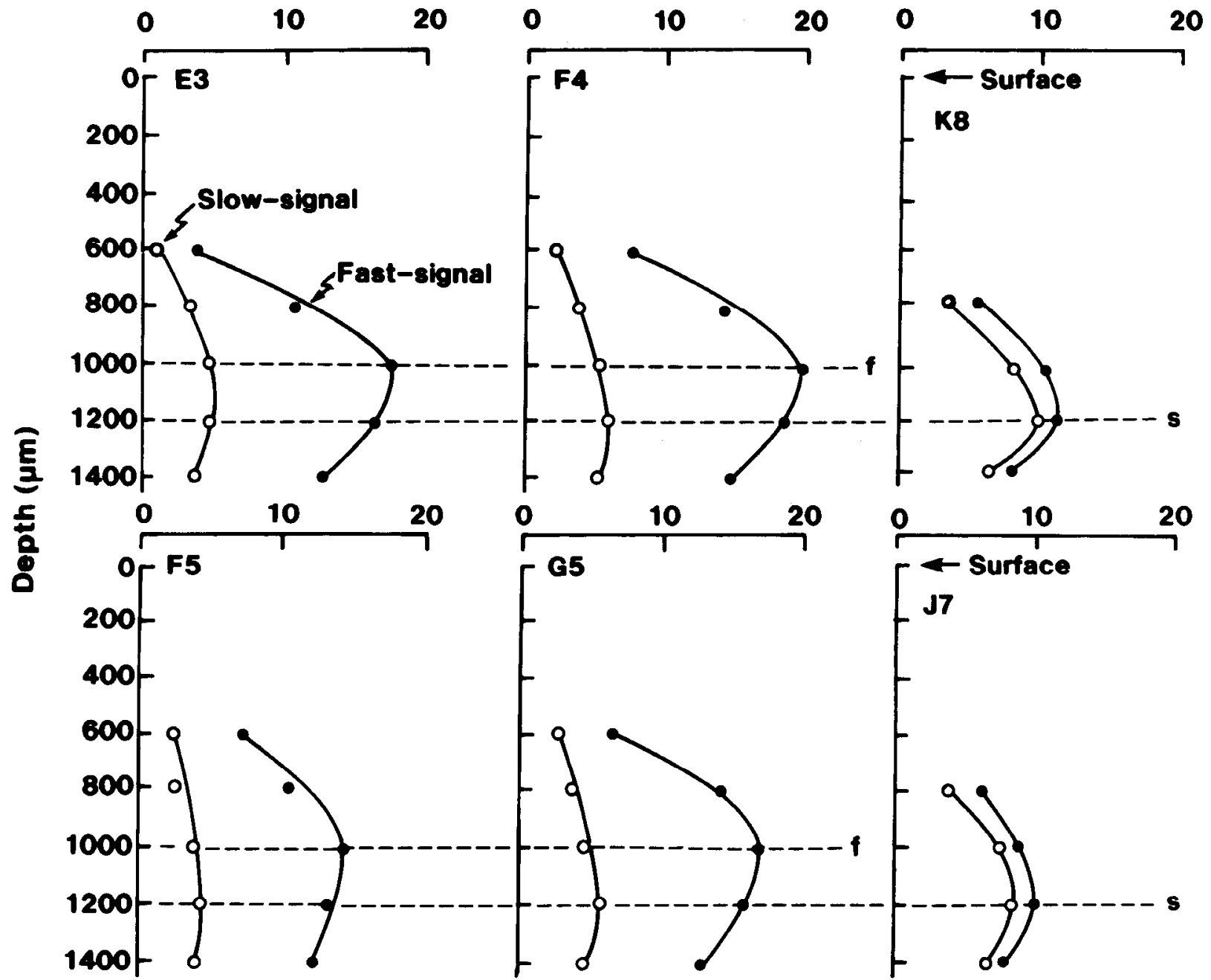

Figure 11. Plots of the size of the vagal response signals versus focal positions for selected detectors. The horizontal dashed lines with label $f$ are placed at the maximum values of the curves for the fast spike-like signal, and the dashed lines with label $s$ are placed at the maximum values of the curves for the slow signal. Other indications are the same as in Figure 5.

teristics, we concluded that the slow signal reflects glutaminergic excitatory postsynaptic potentials within the nucleus of the tractus solitarius (see Discussion). The present results also indicate that the initial phase of the postsynaptic potential is mediated by a non-NMDA-receptor, while the later phase is mediated by the NMDA-receptor.

\section{Comparison with vagal response}

Figure 9 shows an example of the original optical recordings of vagal responses from an intact $8 \mathrm{~d}$ embryonic brainstem preparation (the same preparation as that shown in Fig. 2). When a brief stimulating square current pulse was applied to the vagus nerve, fast spike-like and slow signals were evoked in a concentrated area around the level of the vagus nerve. From such an intact recording alone, it is impossible to obtain information about the three-dimensional distribution of the response area.

We have previously recorded vagal responses from thinner slice preparations at the level of the vagus nerve (Kamino et al. 1989b, 1990; Komuro et al., 1991; Momose-Sato et al., 1991). In the present work, we carried out optical sectioning recordings of the vagal response in thick-slice preparations, and we compared the results with those of the glossopharyngeal response.

Figure 10 illustrates a series of optical sectioning recordings from the same slice preparation as that shown in Figure 4. The experimental protocol was also the same as that in the glossopharyngeal experiment. The size of the evoked optical signal depended on the depth of the focal plane. In Figure 11, the sizes of the fast spike-like and slow signals are plotted against the depth of the focal plane. As expected from the recordings from intact preparations, the patterns of the curves were different from those for the glossopharyngeal responses: quasiparabolic curves having a maximum value near the focal position of $1000 \mu \mathrm{m}$ for the fast signals were obtained from medial regions (E3, F5, $\mathrm{F} 4$, and G5), and $1200 \mu \mathrm{m}$ for the fast signals from lateral regions (K8 and J7). All the slow signals had their peaks at 1200 $\mu \mathrm{m}$.

As for the glossopharyngeal recording, the contour line maps for each section are shown in Figure 12. As previously discussed in experiments concerning the vagal responses (Komuro et al., 1991), the fast spike-like signal is considered to contain two 
N.X/8-day
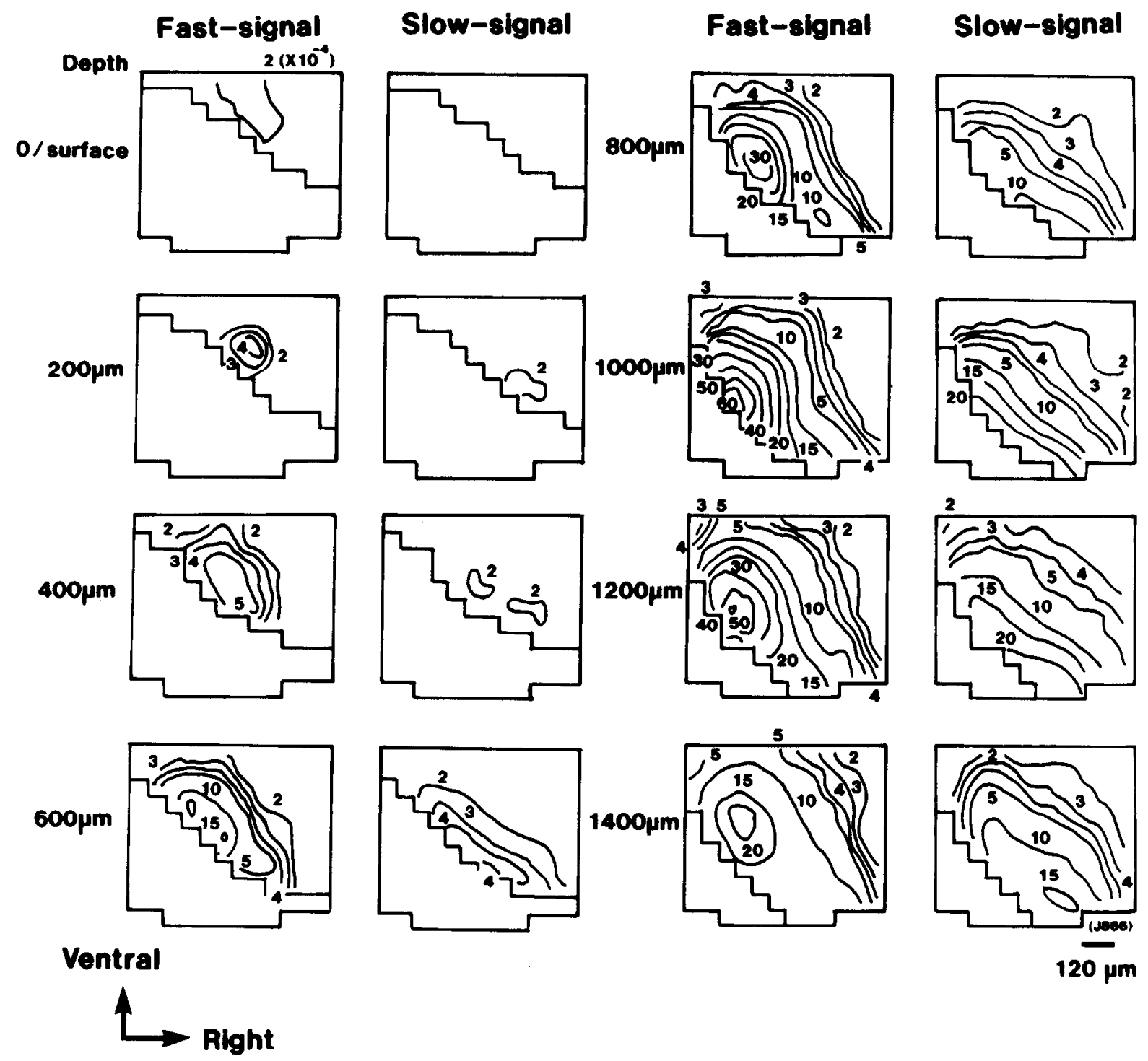

Figure 12. Contour line maps constructed from the recordings shown in Figure 10.

components; one originating in the antidromic action potential in the motor neurons and the other from the orthodromic action potential in the terminal of the sensory nerve. Although it is technically difficult to separate the two components, we assumed that the fast spike-like signal is generated mainly by the antidromic action potential in the motoncurons and that the component related to the orthodromic action potential in the sensory nerve terminals is relatively small (see also Komuro et al., 1991). Therefore, we concluded that the peak-size area and its surrounding of the fast spike-like signal corresponded to the dorsal motor nucleus of the vagus nerve, and that the slow signal area corresponded to the nucleus of the tractus solitarius.

The relative three-dimensional positions of the maximum glossopharyngeal and vagal response areas are compared in Figure 13. Also, we have reconstructed the three-dimensional maps of glossopharyngeal and vagal response areas in an $8 \mathrm{~d}$ old embryonic brainstem preparation which was used in Figures 4 and 10. The results are shown in Figure 14.

\section{Discussion}

In the present report, we describe the first attempt at optical sectioning and multiple-site optical recording to assess the threedimensional functional architecture of the glossopharyngeal and vagal response areas in early embryonic chick brainstem preparations.

Although the results obtained were purely phenomenological, the optical sectioning performance of the present experiments was such that we could obtain reliable signal maps of optical sections from $1400-1600 \mu \mathrm{m}$ thick whole-mount slice preparations dissected from early embryonic brainstems (Figs. 4, 10). The success of the optical sectioning recording resulted from the fact that the early embryonic brainstems that we used have a histologically loose structure with immature connective tissues. Thus, the low opacity allowed the incident light to pass through the slice preparation and the dye (a merocyanine-rhodanine dye, NK2761) that we used stained neurons relatively well in the deeper regions of the preparation. However, the present experi- 


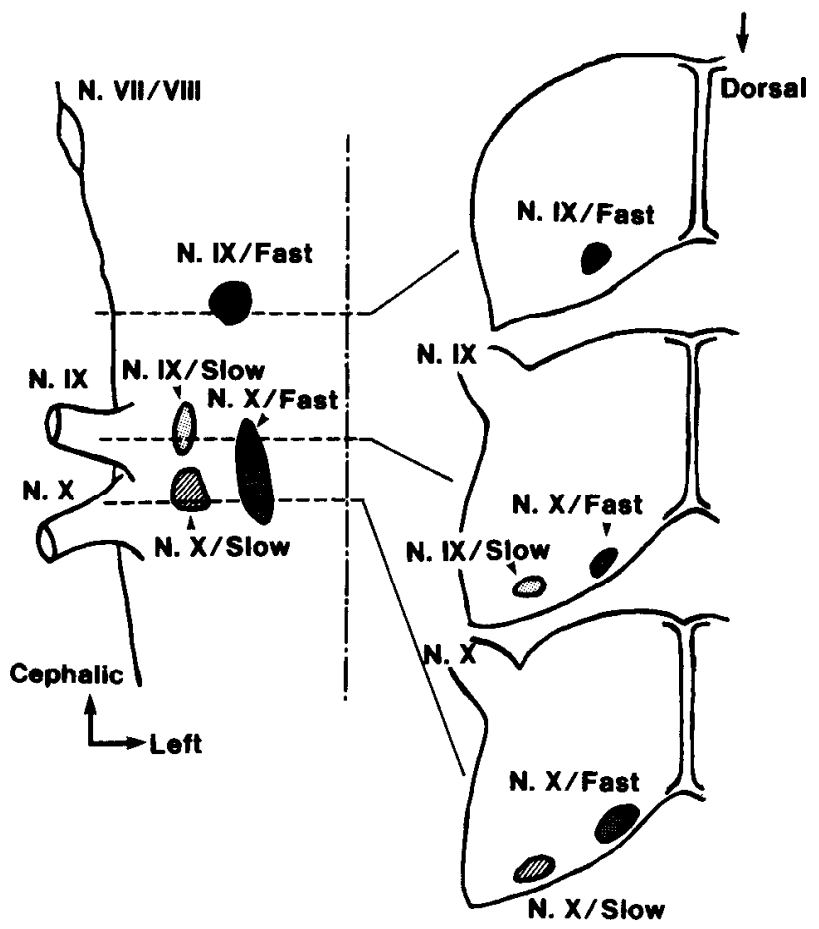

Figure 13. Comparison of the peak-size areas of the glossopharyngeal fast spike-like (N.IX/Fast) and slow (N.IX/Slow) signals and the vagal fast spike-like $(N . X / F$ ast $)$ and slow $(N . X / S l o w)$ signals, in the cephaliccaudal plane and the dorsal-ventral plane. The glossopharyngeal fast spike-like signal area corresponds to the nucleus of the glossopharyngeal nerve (nucleus nervi glossopharyngei), vagal fast spike-like signal area corresponds to the dorsal motor nucleus of the vagus nerve, and both the glossopharyngeal and vagal slow signal areas corresponds to the nucleus of the tractus solitarius.

ments have been associated with several problems to be discussed and examined in more detail.

The availability of three-dimensional imaging procedures for the light microscope (Agard and Sedat, 1983; Agard, 1984; Macias Garza et al., 1989; Hiraoka et al., 1990) has already suggested that optical serial sectioning of voltage-sensitive dye signals through a tissue can be used to determine the three-dimensional spatial structure of the electrical activity (Grinvald et al., 1984, 1988; Cohen and Lesher, 1986; Yae et al., 1992). Threedimensional microscopic data are collected as a series of twodimensional signal images recorded at different focal planes, so that each of these signal images contains not only in-focus information from the positions around the focal plane, but also out-of-focus contributions from the remainder of the preparation: the results of optical sectioning measurements result in a distorted view of the spatial distribution of the signal. The amount of optical distortion depends on several factors including the amount of defocus, the numerical aperture of the objective lens and light scattering of the tissue (Salzberg et al., 1977; Orbach and Cohen, 1983; Agard, 1984; Macias Garza et al., 1989).

Yae et al. (1992) carried out theoretical and experimental studies on the point-spread function for fluorescent voltage-sensitive dye recording using video camera imaging of the cerebellar cortex rather than the photodiode-recording signal size. For the optical serial sectioning/voltagc-scnsitive dyc signals detected by a photodiode array, the size of the observed signal would contain the summation of the blurred "signal sizes" from the defocus positions. Therefore, to assess the true signal size, it is necessary to derive the defocus blurring function for the size of the signals from the defocus positions. However, it is difficult to do the mathematical deconvolution, since there are many factors and parameters to be considered. We have not yet obtained a reliable method to determine the defocus blurring function and pointspread function for optical recording, using a photodiode array, of transmitted light signals from a thick-slice preparation stained with voltage-sensitive dyes. Therefore, it is difficult to obtain compensated maps, at present. Further theoretical studies and modeling experiments are required.

To determine the resolution in the $\mathrm{z}$-axis in the barnacle circumesophageal ganglion, Salzberg et al. (1977) measured the size of the optical signal when a neuron was moved both above and below the focal plane, and found that the signal size was reduced by $50 \%$ when the neuron was moved $\pm 300 \mu \mathrm{m}$ out of focus. Empirically, their results suggest that, in the present recordings, signals detected from any given focal plane may contain blurred signals from positions approximately $\pm 400 \mu \mathrm{m}$ from the focal plane. (This is a very approximate estimation, since the scattering properties of the embryonic brainstem slices used in the present study cannot legitimately be compared with those of the barnacle ganglion.)

Light-scattering can limit the spatial resolution of an optical measurement (Orbach and Cohen, 1983), and substantial light scattering would also reduce the signal-to-noise ratio by spreading the light that would otherwise reach a single detector over many detectors. Thus, in the present study, one must consider the boundary of the response area to be blurred; the present approach is a first approximation, and a first quantitative step in $\mathrm{z}$-axis (depth) resolution of voltage-sensitive dye-dependent optical signal recording. More detailed theoretical and experimental approaches, including the determination of defocus blurring functions for the preparations of interest, are required.

Although there are serious limitations to the present optical sectioning recording and the data processing, the results presented here suggest several conclusions that may be of significance for the developmental neurobiology of the vertebrate brainstem.

The basic phenomenological results concerning the glossopharyngeal response area can be summarized as follows. (1) Two different response areas to glossopharyngeal stimulus are identified in the $7 \mathrm{~d}$ and $8 \mathrm{~d}$ old embryonic brainstems: one is located in the dorsal/lateral region near the level of the glossopharyngeal nerve (area I), and the other is located in the somewhat dorsal/medial region near the level of about $400-500 \mu \mathrm{m}$ from the root of the glossopharyngeal nerve (area II); (2) area I partially overlaps the area of the vagus nerve nucleus of the tractus solitarius. In addition, the fast spike-like signals detected from area I are often associated with a delayed slow long-lasting signal, whereas only fast spike-like signals are detected from area II. Similarly to the vagal evoked slow signal, the glossopharyngeal slow signal is also related to a glutaminergic excitatory postsynaptic potential.

The glossopharyngeal nerve bundle contains both motor and sensory nerve fibers. Therefore, in the present study, the applied stimulating current was orthodromic for the sensory neurons, and it was antidromic for the motor neurons. Based on the characteristics of the maps (Figs. 3, 6) and the curves (Fig. 5), the fast spike-like signals detected from area II are considered to be equivalent to the antidromic action potentials evoked in the motor neurons, and the fast spike-signals from area I are considered to be the orthodromic action potentials in the terminals of the 


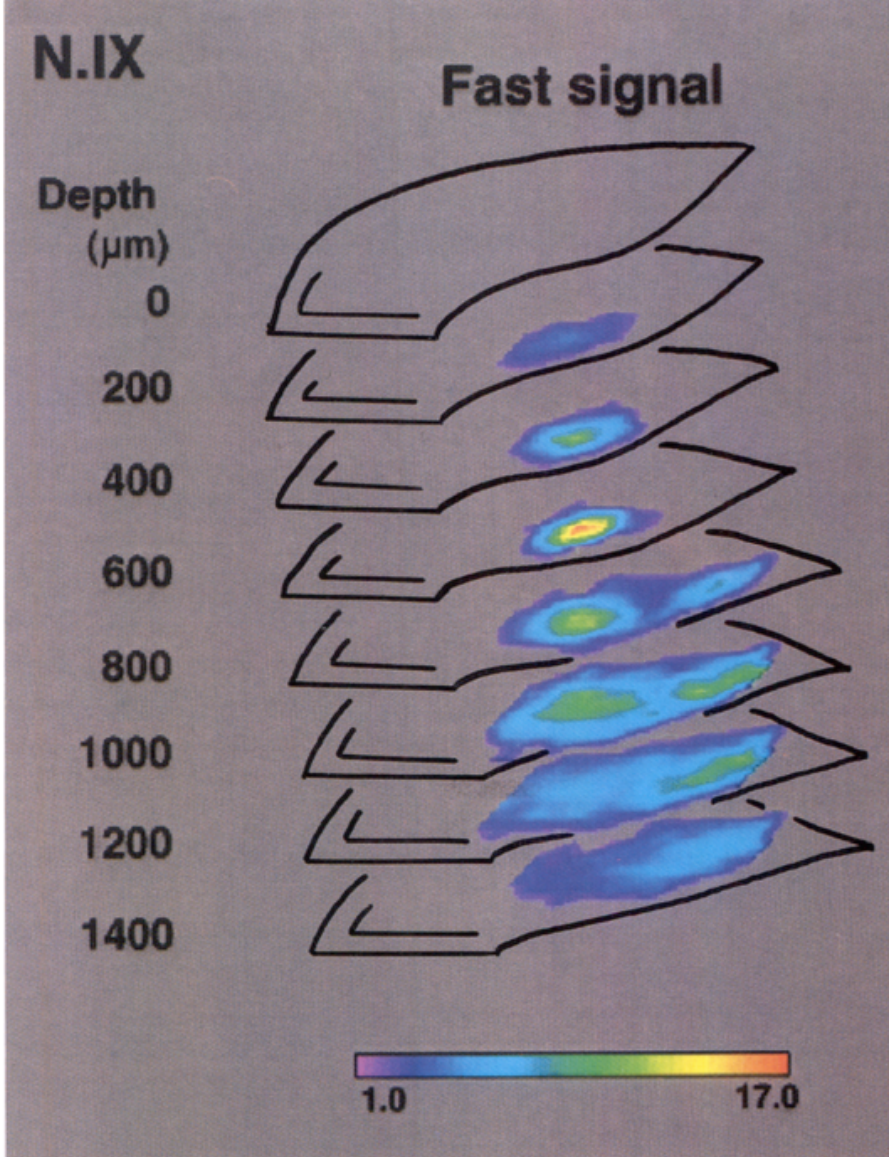

N.X

Fast signal

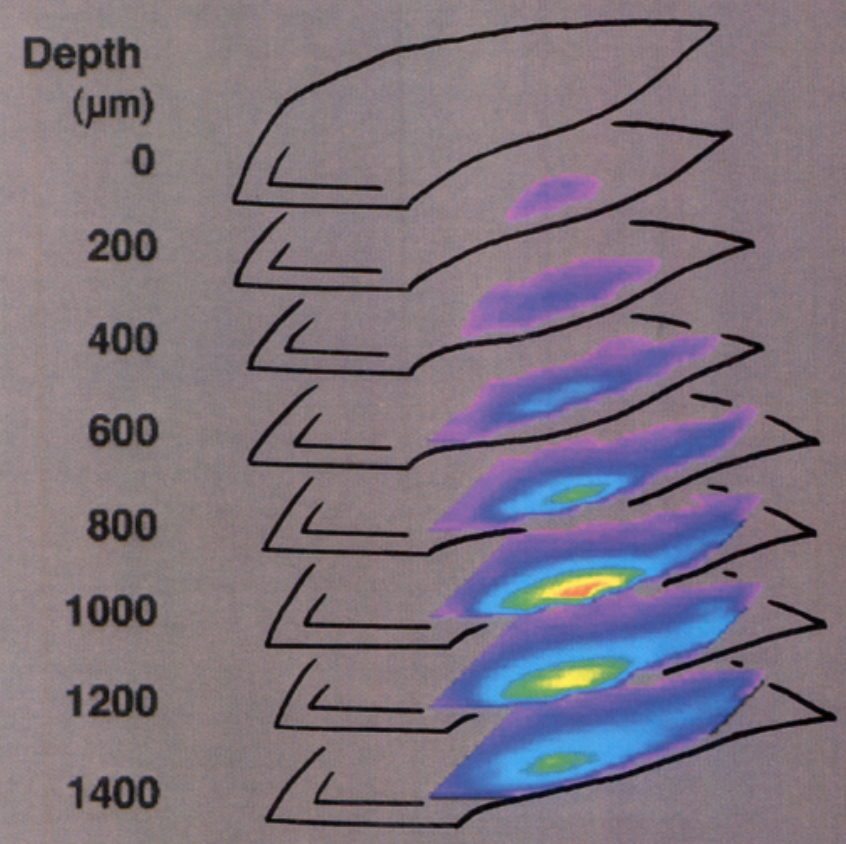

Cephalic

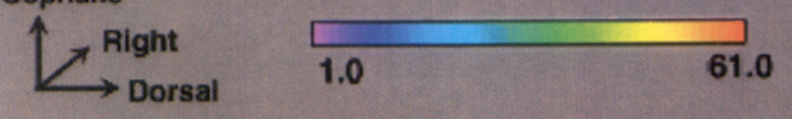

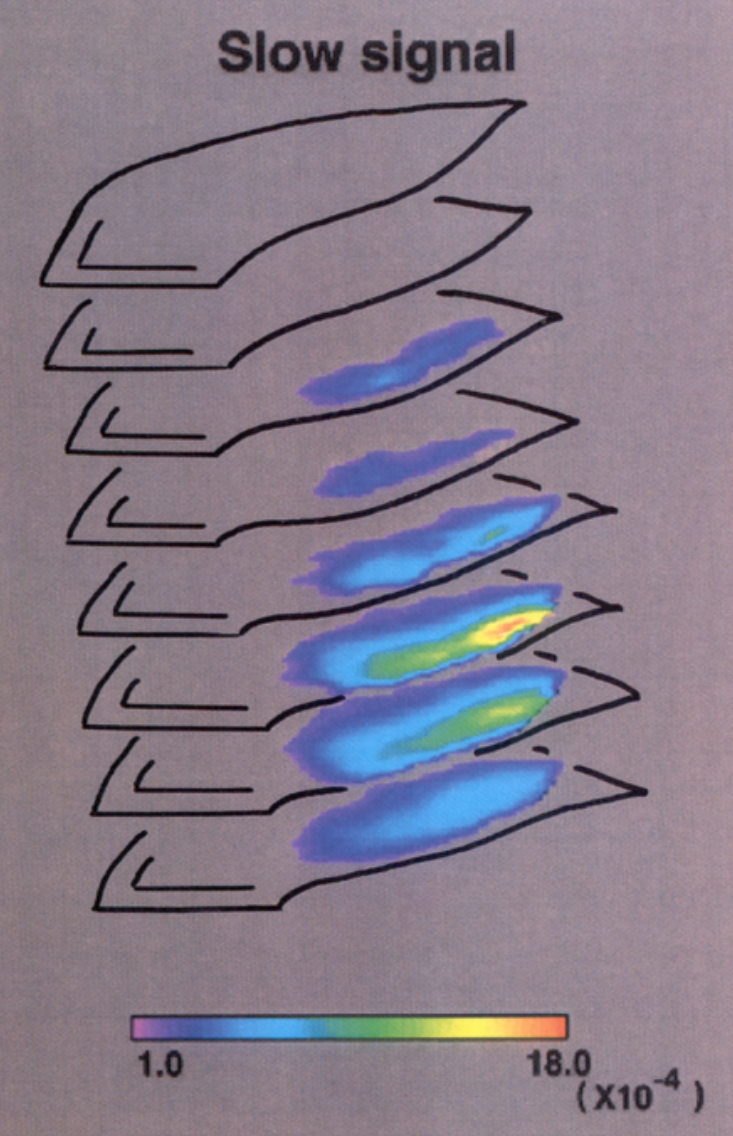

Slow signal
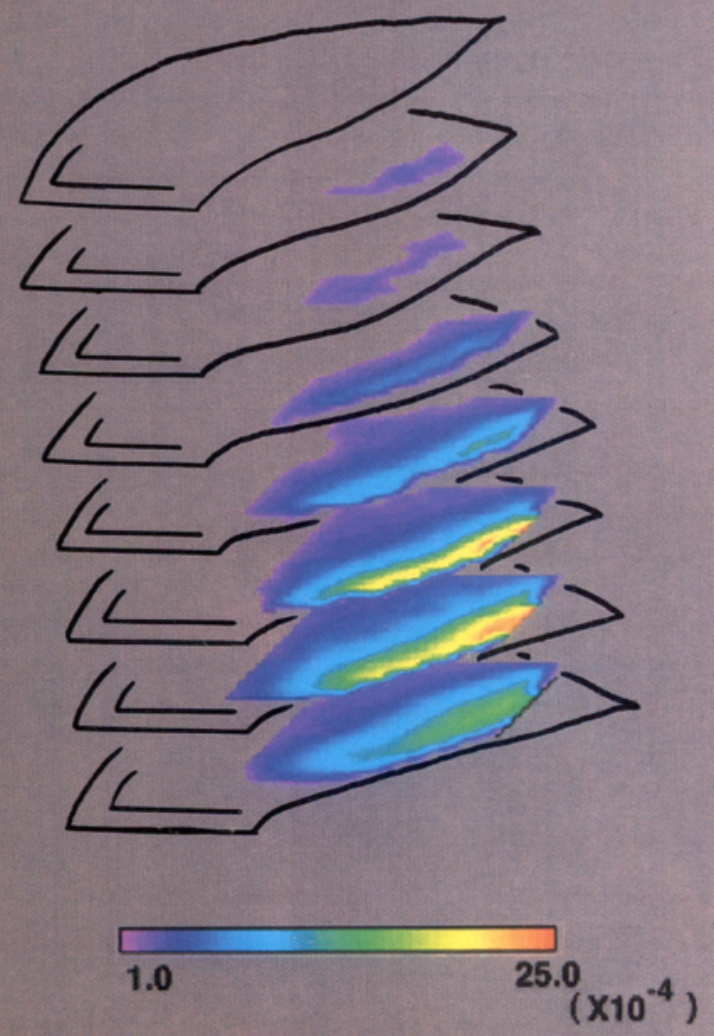
sensory neurons. From these phenomenological observations, we conclude that area I for the glossopharyngeal response corresponds to the nucleus of the tractus solitarius, and that area II reveals the nucleus of the glossopharyngeal nerve (nucleus nervi glossopharyngei; Breazile, 1979). This feature was revealed for the first time in the present study using optical sectioning volt age-sensitive dye recording.

In the optical technique for monitoring membrane potential changes, the linearity of the optical signal with changes in membrane potential has been established in other preparations (Cohen and Salzberg, 1978), and it has also been assumed that the fractional signal size is proportional to the magnitude of the mernbrane potential changes in each cell and process, and to the number and membrane area of activated neural elements within the field detected optically by one photodiode under conditions where the amount of dye bound to the membrane is uniform (Obaid et al., 1985; Orbach et al., 1985; Kamino et al., 1989a; Kamino, 1991). In the present study, the background intensities of transmitted light from the stained preparation were nearly constant from pixel to pixel/from focal plane to focal plane, and the fractional sizes of the displayed signals were normalized by dividing by the background intensities. Thus, we feel justified in assuming (1) that the amplitude of the fast spike-like signal detected from area II (corresponding to the nucleus of the glossopharyngeal nerve) is proportional to the number, size, fraction of membrane area, and action potential activity of activated motor neurons imaged onto one photodiode, (2) that the amplitude of the fast spike-like signal detected from area I (corresponding to the nucleus of the tractus solitarius) is proportional to the number, size, fraction of membrane area, and action potential activity of the activated sensory nerve terminals, and (3) that the amplitude of the slow signal detected from area $I$ is proportional to the number, size, fraction of membrane area, and membrane potential activity of postsynaptic neurons.

According to these assumptions, the spatial distribution pattern of the optical signals reflects the spatial paltern of neural activity as a function of the membrane area of the activated cells and the magnitude of the membrane potential change in each activated cell within the optical receptive field of one photodiode. Therefore, it seems possible, or even likely, (1) that the nucleus of the glossopharyngeal nerve is organized in a threedimensionally layered pattern with regard to the related motor neurons, with a central core composed of a population of motor neurons having the highest activity, and (2) that the nucleus of the tractus solitarius is similarly organized in a three-dimensionally layered pattern with the related sensory neurons/synapses, and a population of the neurons/synapses having the highest activity again making a central core. The motor nuclei related to vagus and glossopharyngeal nerves were observed to be located separately in both $7 \mathrm{~d}$ and $8 \mathrm{~d}$ old brainstems (see Fig. 13). Furthermore, the findings of the present study reveal that the central core of the glossopharyngeal nucleus of the tractus solitarius is located separately from the central core of the vagal nucleus of the tractus solitarius (Fig. 13): the glossopharyngeal nucleus is located at the level of the root of the glossopharyngeal nerve, and the vagal nucleus is located at the level of the root of the vagus nerve. This evidence suggests a correlation between the functional organization of both the glossopharyngeal and vagal nuclei and rhombomeres during early development of the chick hindbrain (Lumsden and Keynes, 1989).

Morphologically, in the adult avian brainstem, it is known that a portion of the nucleus of the glossopharyngeal nerve is associated with the dorsal motor nucleus of the vagus nerve (Breazile, 1979). However, in both the $7 \mathrm{~d}$ and $8 \mathrm{~d}$ old embryonic brainstems, the nucleus of the glossopharyngeal nerve and the dorsal motor nucleus of the vagus nerve are separated as illustrated in Figure 13. Furthermore, several investigators have divided the nucleus of the glossopharyngeal nerve into dorsal and ventral parts, and the dorsal part is associated with the vagus nucleus (Kuhlenbeck, 1975). However, no evidence for this scparation was observed in the present study.

\section{References}

Agard DA (1984) Optical sectioning microscopy: cellular architecture in three dimensions. Annu Rev Biophys Bioeng 13:191-219.

Agard DA, Sedat JW (1983) Three-dimensional architecture of a polytene nucleus. Nature 302:676-681.

Albowitz B, Kuhnt U (1993) Evoked changes of membrane potential in guinea pig sensory neocortical slices; an analysis with voltagesensitive dyes and a fast optical recording method. Exp Brain Res 93:213-225.

Breazile JE (1979) Systema nervosum centrale. In: Nomina anatomica avium: an annotated anatomical dictionary of birds (Baumel JJ, King AS, Lucas AM, Breazile JE, Evans HE, eds), pp 417-472. New York: Academic.

Cinelli AR, Salzberg BM (1990) Multiple site optical recording of transmembrane voltage (MSORTV), single-unit recordings, and evoked field potentials from the olfactory bulb of skate (Raja erinacea). J Neurophysiol 64:1767-1790.

Cinelli AR, Salzberg BM (1992) Dendritic origin of late events in optical recordings from salamander olfactory bulb. $J$ Neurophysiol 68:786-806.

Cohen LB, Lesher S (1986) Optical monitoring of membrane potential: methods of multisite optical measurement. In: Optical methods in cell physiology (De Weer P, Salzberg BM, eds), pp 71-99. New York: Wiley.

Cohen LB, Salzberg BM (1978) Optical measurement of membrane potential. Rev Physiol Biochem Pharmacol 83:35-88.

Erhardt A, Zinser G, Komitowski D, Bille J (1985) Reconstructing 3-D light-microscopic images by digital image processing. Appl Optics 24:194-200.

Fukunishi K, Murai N, Uno H (1992) Dynamic characteristics of the auditory cortex of guinea pigs observed with multichannel optical recording. Biol Cybern 67:501-509.

Grinvald A, Cohen LB, Lesher S, Boyle MB (1981) Simultaneous optical monitoring of activity of many neurons in invertebrate ganglia using a 124-element photodiode array. J Neurophysiol 45:829-840.

Grinvald A, Anglister L, Freeman JA, Hildesheim R, Manker A (1984) Real-time optical imaging of naturally evoked electrical activity in intact frog brain. Nature 308:848-850.

Grinvald A, Frostig RD, Lieke E, Hildesheim R (1988) Optical imaging of neuronal activity. Physiol Rev 68:1285-1366.

Hiraoka Y, Sedat JW, Agard DA (1990) Determination of three-dimensional imaging properties of a light microscope system: partial confocal behavior in epifluorescence microscopy. Biophys J 57:325-333.

Kamino K (1991) Optical approaches to ontogeny of electrical activity and related functional organization during early heart development. Physiol Rev 71:53-91

Kamino K, Hirota A, Fujii S (1981) Localization of pacemaking activity in early embryonic heart monitored using a voltage-sensitive dye. Nature 290:595-597.

Kamino K, Hirota A, Komuro H (1989a) Optical indications of elec-

Figure 14. Three-dimensional reconstructions of glossopharyngeal (upper panels, N.IX) and vagal (lower panels, N.X) response areas in an $8 \mathrm{~d}$ old embryonic brainstem preparation; four panels were computationally reconstructed from the same preparation shown in Figures 4 and 10 . Left panels show the fast-spike-like signal response areas, and right panels show the slow response areas. 
trical activity and excitation-contraction coupling in the early embryonic heart. Adv Biophys 25:45-93.

Kamino K, Katoh Y, Komuro H, Sato K (1989b) Multiple-site optical monitoring of neuronal activity evoked by vagus nerve stimulation in the embryonic chick brain stem. J Physiol (Lond) 409:263-283.

Kamino K, Komuro H, Sakai T, Sato K (1990) Optical assessment of spatially ordered patterns of neural response to vagal stimulation in the early embryonic chick brainstem. Neurosci Res 8:255-271.

Kauer JS, Senseman DM, Cohen LB (1987) Odor-elicited activity monitored simultaneously from 124 regions of the salamander olfactory bulb using a voltage-sensitive dye. Brain Res 418:255-261.

Komuro H, Sakai T, Momose-Sato Y, Hirota A, Kamino K (1991) Optical detection of postsynaptic potentials evoked by vagal stimulation in the early embryonic chick brain stem slice. J Physiol (Lond) 442: 631-648.

Kuhlenbeck H (1975) The central nervous system of vertebrates, Vol 4, Spinal cord and denterencephalon. New York: Karger.

Litaudon P, Cattarelli M (1992) Origin of the in vivo rat piriform cortex activity recorded with voltage-sensitive dyes: comparison of the optical signals and the field potentials. Brain Res 594:171-175.

London JA, Zecevic D, Cohen LB (1987) Simultaneous optical recording of activity during feeding in Navanax. J Neurosci 7:649-661.

Lumsden A, Keynes R (1989) Segmental patterns of neuronal development in the chick hindbrain. Nature 337:424-428.

Macias Garza F, Diller KR, Bovik AC, Aggarwal SJ, Aggarwal JK (1989) Improvement in the resolution of three-dimensional data sets collected using optical serial sectioning. J Microsc 153:205-221.

Momose-Sato Y, Sakai T, Komuro H, Hirota A, Kamino K (1991) Optical mapping of the early development of the response pattern to vagal stimulation in embryonic chick brain stem. J Physiol (Lond) 442:649-668.
Momose-Sato Y, Sato K, Sakai T, Hirota A, Kamino K (1993) A trial of optical multiple-site/sectioning recording of neural responses to glossopharyngeal stimulus in the early embryonic brainstem. Neurosci Res [Suppl] 18:S29.

Obaid AL, Orkand RK, Gainer H, Salzberg BM (1985) Active calcium responses recorded optically from nerve terminals of the frog neurohypophysis. J Gen Physiol 85:481-489.

Orbach HS, Cohen LB (1983) Optical monitoring of activity from many areas of the in vitro and in vivo salamander olfactory bulb: a new method for studying functional organization in the vertebrate central nervous system. J Neurosci 3:2251-2262.

Orbach HS, Cohen LB, Grinvald A (1985) Optical mapping of electrical activity in rat somatosensory and visual cortex. $J$ Neurosci 5:1886-1895.

Sakai T, Sato K, Momose-Sato Y, Hirota A, Kamino K (1993) Optical multiple-site/sectioning recording of glossopharyngeal responses in the early embryonic brainstem. Soc Neurosci Abstr 19:644.

Salzberg BM, Grinvald A, Cohen LB, Davila HV, Ross WN (1977) Optical recording of neuronal activity in an invertebrate central nervous system: simultaneous monitoring of several neurons. J Neurophysiol 40:1281-1291.

Salzberg BM, Obaid AL, Senseman DM, Gainer H (1983) Optical recording of action potentials from vertebrate nerve terminals using potentiometric probes provides evidence for sodium and calcium components. Nature 306:36-40.

Taniguchi I, Nasu M (1993) Spatio-temporal representation of sound intensity in the guinca pig auditory cortex observed by optical recording. Neurosci Lett 151:178-181.

Yae H, Elias SA, Ebner TJ (1992) Deblurring of 3-dimensional patterns of evoked rat cerebellar cortical activity: a study using voltage-sensitive dyes. J Neurosci Methods 42:195-209. 